\title{
Network Configurations for Seamless Support of CDMA Soft Handoffs Between Cell Clusters
}

\author{
Bing H. Cheung and Victor C. M. Leung, Member, IEEE
}

\begin{abstract}
Current code division multiple access (CDMA) networks do not support soft handoffs between cell clusters serviced by different mobile switching centers (MSC's). Three novel network configurations are proposed to overcome this limitation. Configurations I and II employ crosslinks to allow diversity combining of signals. This is accomplished in Configuration III by linking the modified MSC's serving adjacent cell clusters. In Configuration I, a scheme which reduces the handoff blocking probability by lengthening the handoff transition time is proposed. Configuration II expands overlapping regions of adjacent cell clusters, thus enabling the use of different intercluster handoff regions for different handoff directions to prevent oscillations which may occur in Configuration I, while reducing the necessary number of intercluster handoffs. Configuration III fully shares the trunks to support the highest number of calls among all three configurations. A new mobility model for microcells is developed to enable dimensioning of the trunk groups in each configuration. For Configurations I and II, the optimal partitions of trunk groups into direct and crosslinks are determined by mathematical analyses and computer simulations. For Configuration III, the optimal number of trunks required to interconnect adjacent MSC's is investigated by computer simulations. The new call and handoff blocking probabilities in all of these configurations are compared.
\end{abstract}

Index Terms-Code division multi-access, communication system planning, land mobile radio cellular systems, personal communication networks.

\section{INTRODUCTION}

C ODE division multiple access (CDMA) is an emerging air interface standard for digital cellular systems (e.g., the TIA IS-95 standard) and future personal communication networks (PCN's). One of many distinct features of CDMA is the support of soft handoffs between adjacent cells for mobile terminals (MT's) crossing cell boundaries [1]-[3]. Soft handoffs allow MT's in handoff regions to communicate with old and new base stations (BS's) simultaneously, enabling diversity combining of signals to minimize signal degradations and disruptions. Because of the "make before break" switching function of radio links, soft handoffs are inherently seamless, and minimize the undesirable "ping-pong" phenomenon of

Manuscript received September 3, 1996; revised March 24, 1997. This work was supported by a grant from the Canadian Institute for Telecommunications Research under the NCE Program of the Canadian Government. This work is based in part on a paper presented at IEEE ICUPC'96, Boston, MA.

B. H. Cheung was with the Department of Electrical Engineering, University of British Columbia, Vancouver, B.C. V6T 1Z4, Canada. He is now with the Wireless Networks Planning Group, NORTEL, Ottawa, Ont., Canada.

V. C. M. Leung is with the Department of Electrical Engineering, University of British Columbia, Vancouver, B.C. V6T 1Z4, Canada.

Publisher Item Identifier S 0733-8716(97)05857-5. back-and-forth handoffs between two adjacent cells in conventional hard handoffs.

In PCN's employing microcells, soft handoffs between two cells within a cluster of microcells connected to the same mobile switching center (MSC) are readily achieved by diversity combining uplink signals from the two adjacent BS's at the common MSC and coherently combining the corresponding downlink signals by a RAKE receiver at the MT [4]-[9]. This handoff process is managed entirely by the common MSC without the involvement of the backbone intelligent network (IN), and it does not require the rerouting of network connections.

As PCN's become ubiquitous, it is necessary to extend the same handoff service between microcell clusters connected to different MSC's, so that a uniform quality of service can be provided to the users. This service requires the capabilities of fast and seamless rerouting of calls between MSC's over the backbone network, and soft handoffs between BS's in adjacent cell clusters. While solutions for rerouting ATM/B-ISDN network connections between different MSC's have recently been proposed [10]-[12], the support of soft handoffs between adjacent cell clusters has not received much consideration in the literature, and is the main contribution of this paper. In particular, we propose and evaluate novel configurations of BS-MSC and MSC-MSC links and appropriate handoff procedures to enable CDMA soft handoffs for MT's crossing cell-cluster boundaries. Link capacities required for these configurations to support the maximum traffic level possible in each microcell are also analyzed. The analyses require the derivation of handoff rates and channel holding times using some mobility model. Whereas traffic statistics and mobility models for macrocells have been widely discussed in the literature [13]-[15], the mobility model for microcells has received less attention [16], and a model suitable for the derivation of the above traffic statistics is not available. A secondary contribution of this paper is the development of a mobility model of vehicular users in stop-and-go traffic moving through the microcells covering the streets in a city center. Although we have not done so in this paper, the mobility model is easily extended to account for pedestrian users [17].

The outline of this paper is as follows. Following this introduction, Section II presents three novel network configurations which support CDMA soft handoffs between microcells in adjacent cell clusters. Section III proposes a new mobility model for MT's traveling through microcells in a city center. This facilitates analyses for the dimensioning and partitioning 


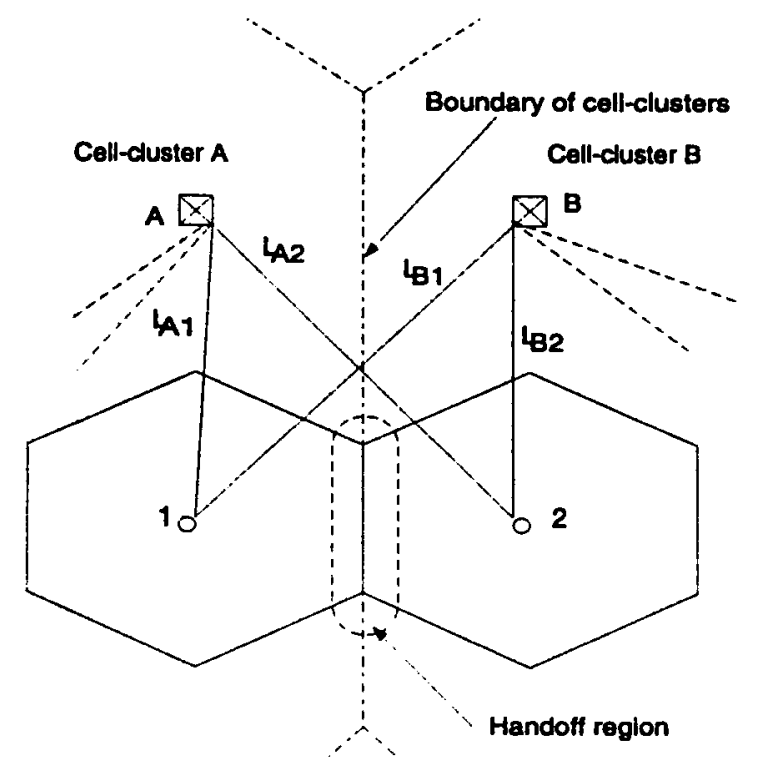

Microcell O Base station

\section{Mobile switching centre}

Fig. 1. Network Configuration I.

of trunk groups in the respective network configurations in Section IV. Finally, Section V draws some conclusions.

\section{NETWORK CONFIGURATIONS}

Three novel network configurations are proposed to support CDMA soft handoffs between clusters of microcells connected to different MSC's. Configurations I and II employ crosslinks between the BS's of boundary cells and MSC's serving the adjacent cell clusters. In Configuration III, the MSC's of different cell clusters are directly connected to enhance trunking efficiency.

\section{A. Configuration I}

In Configuration I, each pair of cells at the edge of two adjacent cell clusters are crosslinked with the MSC's serving the opposing cell clusters. This is illustrated in Fig. 1, where adjacent cells 1 and 2 are at the boundary of two neighboring cell clusters, direct links $L_{A 1}$ and $L_{B 2}$ connect MSC's $A$ and $B$ to cells 1 and 2, respectively, and crosslinks $L_{A 2}$ and $L_{B 1}$ interconnect cells 2 and 1 to MSC's $A$ and $B$, respectively. Addition of the crosslinks enables soft handoffs between the two cells by allowing diversity combining of received signals at the MSC's and MT's during the handoff process. The soft handoff operation between cells 1 and 2 is described below.

Consider an MT moving from cell 1 to 2 . When the MT enters the handoff region at the boundary between the two cells, a CDMA channel is assigned in cell 2 to enable the MT to communicate simultaneously with both BS's 1 and 2. Initially, the MT's call is routed through MSC $A$ and its connections to BS's 1 and 2 via direct link $L_{A 1}$ and crosslink $L_{A 2}$, respectively, and diversity combining occurs at MSC
$A$ for the uplink and at the MT for the downlink. At the same time, network signaling is invoked, possibly through the IN, to set up a new network connection to MSC $B$ for the handoff between the cell clusters. Upon successful connection establishment, an alternate route for the call exists through MSC $B$ and its connections to BS's 1 and 2 via crosslink $L_{B 1}$ and direct link $L_{B 2}$, respectively. At this point, a downlink signal for the MT is available at MSC $B$ from the network, but is not yet forwarded to the MT over the air interface via $L_{B 1}$ and $L_{B 2}$, and an uplink signal from the MT is available at MSC $B$ via $L_{B 1}$ and $L_{B 2}$, but is not yet forwarded to the network. Seamless handoff of network connections is now executed (e.g., using the connection architecture and protocols proposed in [12] or using IN services) to switch from MSC $A$ to MSC $B$ to complete the new connection for the uplink traffic over the network and for the downlink traffic over BS's 1 and 2 via $L_{B 1}$ and $L_{B 2}$, respectively. When the new connection is in full operation, the old network connection to MSC A and the connections from MSC $A$ to BS's 1 and 2 via $L_{A 1}$ and $L_{A 2}$ are dropped. As soon as the signal strength for the MT has reduced below a certain threshold in cell 1 , crosslink $L_{B 1}$ and the corresponding CDMA channel in cell 1 are dropped. This completes the seamless soft handoff operation over the air interface and network, and the MT now communicates exclusively through BS 2 and MSC $B$.

In the above handoff operation, as long as the MT which has moved from the cell 1 to 2 stays in cell 2, communications will not be disrupted regardless of the delays in completing the new handoff connection to MSC $B$ over the network. It is therefore expected that the IN is quite capable of handling the network portion of the handoff between MSC $A$ and MSC $B$. In fact, by delaying the completion of the handoff from MSC $A$ to $B$, possible oscillations between the handoff connections due to the MT moving back and forth across the boundary of the cell clusters can be reduced since the same handoff region is shared by MT's moving from cell 1 to 2 and vice versa. Another valid reason for delaying the handoff between MSC $A$ and $B$ is that bandwidth is not currently available in $L_{B 2}$ to accommodate the handoff. In this case, instead of dropping the call, it can be maintained through $L_{A 2}$ and MSC $A$ until bandwidth becomes available in $L_{B 2}$. This possibility is considered in channel assignment scheme $B$ for trunk dimensioning in Section IV. Note, however, that delays in handoff completion incur penalties in lowering the traffic capacity of each microcell and increasing the capacity required in the crosslinks. Due to these tradeoffs, some oscillations are unavoidable, and these oscillations may increase the signaling traffic in the network. However, using Configuration II presented in the next section, these oscillations can be avoided completely.

\section{B. Configuration II}

To overcome the problems of oscillations, the MT should have moved some distance into the new cell cluster as suggested by [10] before the intercluster handoff is carried out. Configuration II illustrated by Fig. 2 accomplishes this objective. In addition to the microcells at the boundary of 


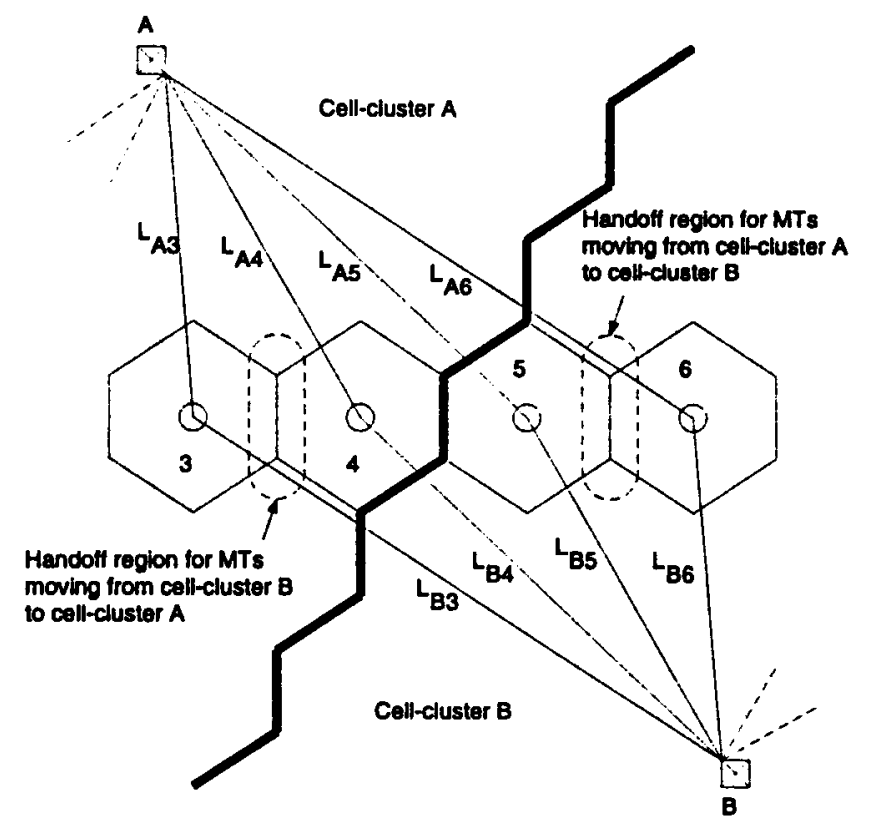

0 Microcell $\bigcirc$ Base station

Mobile switching centre Boundary of cell-elusters

Fig. 2. Network Configuration II.

the cell clusters being crosslinked to the MSC's serving the neighboring cell clusters as in Configuration I, the next layer of cells in the cluster adjacent to the boundary cells is likewise crosslinked to the opposite MSC's. In Fig. 2, MT's moving from cell cluster $A$ to $B$ and those moving from cell cluster $B$ to $A$ have different intercluster handoff regions. An MT belonging to cell cluster $A$, upon moving into cell cluster $B$, delays handing off from MSC $A$ to $B$ until it reaches the handoff region between cells 5 and 6 , after which it is considered to belong to cell cluster $B$. Once the MT belongs to cell cluster $B$, even if it moves from cell 5 back to cell 4 in cell cluster $A$, the handoff is still handled completely by MSC $B$ in the normal manner, and the handoff between MSC's $B$ and $A$ is deferred until the MT crosses the handoff region between cells 4 and 3 . The use of two separate handoff regions completely eliminates the problem of oscillations. Note that MT's originating calls in cells 4 and 5 are considered to belong to cell clusters $A$ and $B$, respectively. The operation of the seamless soft handoff between different MSC's when an MT belonging to one cell cluster enters the handoff region of the opposite cell cluster is as specified previously for Configuration I.

By increasing the complexity of the interconnection network, Configuration II overcomes the problems of oscillating handoffs between the cell clusters. This concept can be extended to include additional layers of cells to be crosslinked between the cell clusters. Effectively, this configuration expands the size of the cell clusters and allows them to overlap, and the delay in executing the more complex handoff procedures between the MSC's after an MT crosses the cell-cluster boundary is determined by the time it takes the MT to go through the overlapped cells. For example, suppose an MT is moving toward cell cluster $B$ from $A$. Handoff between the respective MSC's is not necessary until the MT crosses the boundary of cells 5 and 6 (instead of the boundary of cells 4 and 5 in Configuration I). If this delay can be made long enough relative to the durations of most calls by crosslinking additional layers of cells, the need for handoffs between different MSC's can be significantly reduced. Fig. 3 shows computer simulation results giving the fraction of MSC handoffs avoided among the cluster boundary crossings for two different degrees of overlapping between the cell clusters. Cell $\boldsymbol{a}$ at the boundary of its cell cluster is shown adjacent to different layers of cells in the neighboring cell cluster, which represent the overlapping regions of the cell clusters and are crosslinked to the MSC serving cell $\boldsymbol{a}$ 's cell cluster. When an MT crosses the boundary of the cell clusters from cell $\boldsymbol{a}$, the handoff between MSC's is avoided until the MT moves outside these overlapped cell layers. Movements of the MT's in the simulations follow the mobility model in Section III with stopping probability $p=0.25$. For cell radius $R=500$ $\mathrm{m}$, approximately 80 or $94 \%$ of the potential MSC handoffs can be avoided by using one or two overlapped cell layers, respectively.

\section{Configuration III}

In the above two configurations, four separate trunk groups are involved during soft handoffs. This increases the traffic load and reduces the effective usage of network connections. To overcome these disadvantages, Configuration III as shown in Fig. 4 is proposed as a means to aggregate handoff traffic and improve trunking efficiency. In addition to the direct links between MSC's and BS's, the MSC's of adjacent cell clusters are directly connected by a trunk group shared by all calls engaging in soft handoffs between the two cell clusters. The MSC's need to be modified to enable routing of uplink/downlink diversity signals between adjacent MSC's through these trunks. In comparison with the structure of a typical MSC as described in [18] and [19], the modified MSC requires an additional switching circuit at the front end (where BS's are connected to the MSC) to exchange signals with adjacent modified MSC's.

In Configuration III, since signals from any cell in a cell cluster may be routed from the MSC serving that cell cluster to the MSC serving a neighboring cell cluster, the different intercluster soft handoff methods presented above for Configurations I and II can all be realized. With reference to Fig. 4, the same intercluster handoff method as in Configuration I is realized by selecting the boundary of cells 8 and 9 as the intercluster handoff region. In comparison with Fig. 1, cells 1 and 2 correspond, respectively, to cells 8 and 9 in Fig. 4, and crosslinks $L_{A 2}$ and $L_{B 1}$ are represented, respectively, in Fig. 4 by $L_{B 9}$ and $L_{A 8}$ concatenated with the MSC-MSC link $L_{A B}$. Note that the same connections, $L_{B 9}$ and $L_{A 8}$ in Fig. 4, also serve as the direct links $L_{B 2}$ and $L_{A 1}$, respectively, in Fig. 1. Similarly, to realize the intercluster handoff method used in Configuration II, the handoff region from cluster $A$ to $B$ is between cells 9 and 10 in Fig. 4, and that from 

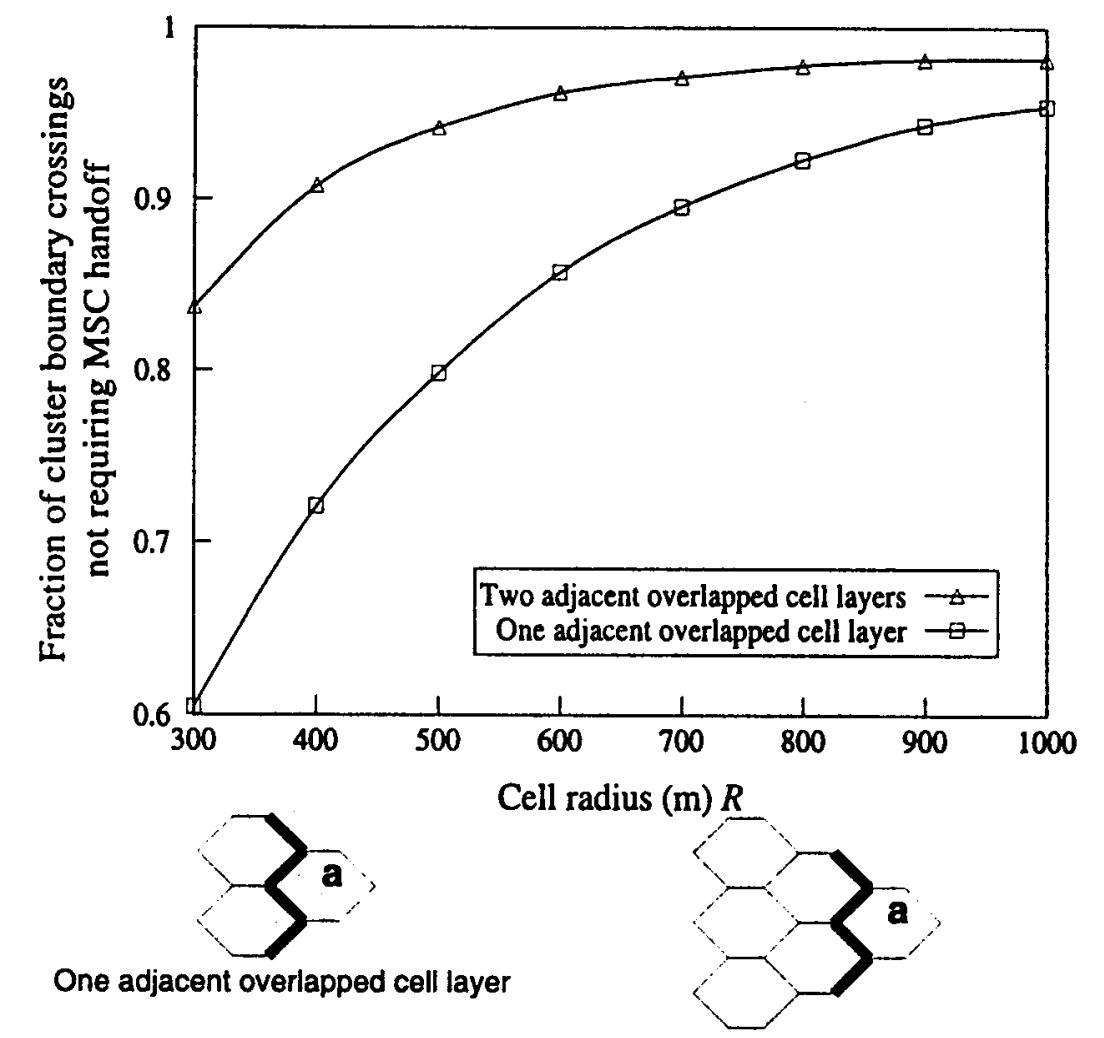

Boundary of cell-clusters

Two adjacent overlapped cell layers

Fig. 3. Effects of different degrees of overlapping at cluster boundary.

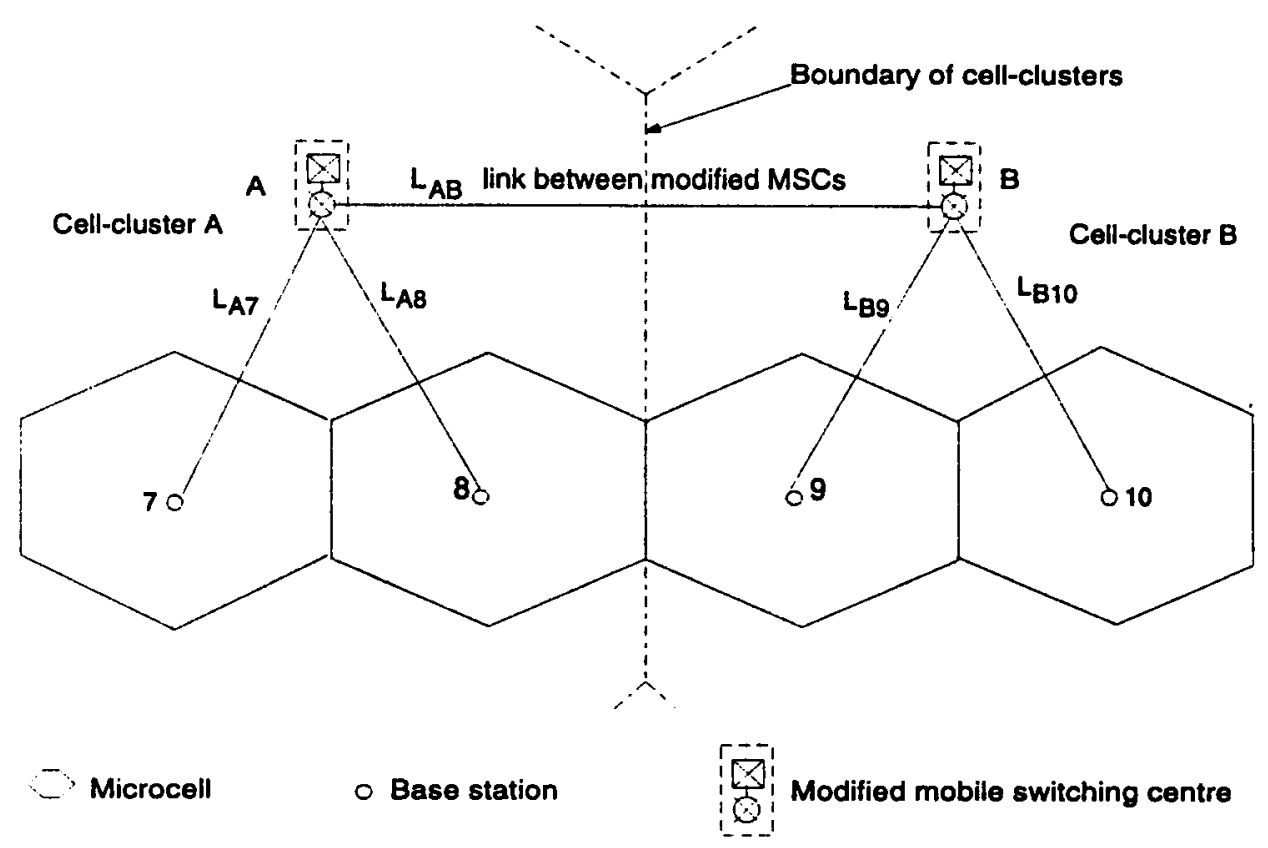

Fig. 4. Network Configuration III.

cluster $B$ to $A$ is between cells 8 and 7 . The crosslinks in Fig. 2 are likewise realized by sharing the respective direct links in concatenation with the MSC-MSC link in Fig. 4. Substituting for the corresponding links, the intercluster soft handoff procedures described previously for Configurations I and II become directly applicable to the respective case for Configuration III.
Because Configuration III allows all channels in the BS-MSC links to be fully shared by new calls and handoff calls, and the MSC-MSC links to be shared by all intercluster handoff calls, an increased number of calls can be accommodated than in Configurations I and II due to improved efficiency. This is verified by analysis, and the results are shown in Section IV-C. 


\section{MOBILITY MODEL}

Before dimensioning the BS-MSC links, the mobility pattern of MT's in microcells needs to be analyzed to enable the channel holding time and handoff rate to be determined. Since a mobility model for microcells suitable for this purpose is not available, a new model needs to be developed. The following mobility model is developed for stop-and-go vehicular traffic through microcells covering the city blocks in an urban center. While it is not considered here, the model is readily modified to account for pedestrian traffic with a lower level of mobility [17].

Consider a specific hexagonal microcell with radius $R$ having a BS located at its center. Assume that MT's are uniformly distributed over a circular region with radius $7 R$ around the BS. The hexagonal cell is surrounded by three circular rings of neighboring cells with radii: $R$ to $3 R, 3 R$ to $5 R$, and $5 R$ to $7 R$. The original location of an MT is represented by its distance $r$ and angle $\theta$ from the specific $\mathrm{BS}$, given by the following distributions:

$$
\begin{aligned}
& f_{r}(r)= \begin{cases}2 r /(7 R)^{2}, & \text { for } 0 \leq r \leq 7 R \\
0, & \text { elsewhere }\end{cases} \\
& f_{\theta}(\theta)= \begin{cases}1 / 2 \pi, & \text { for } 0 \leq \theta \leq 2 \pi \\
0, & \text { elsewhere }\end{cases}
\end{aligned}
$$

The mobility pattern of each MT is as follows. The movements of an MT consist of moving and stopping intervals, consistent with traffic in a city center where the microcell is likely located. Let $p$ be the probability that an MT enters a stopping interval. Thus, with probability $1-p$, the MT enters a moving interval after a stopping or moving interval. Let random variables $T_{m}$ and $T_{s}$ be the duration of each moving and stopping interval, respectively, uniformly distributed in $[0$, $\left.T_{m_{-} \max }\right]$ and $\left[0, T_{s_{-} \max }\right]$. Let random variable $V$ be the speed of an MT in a moving interval, assumed uniformly distributed in $\left[0, V_{\max }\right]$. As appropriate for movements around city blocks in an urban center, the direction of travel is assumed to change before each moving interval with an angle uniformly distributed between $\pm \phi$ relative to the MT's traveling direction in the previous moving interval. Finally, let random variable $T$ be the unencumbered message duration (call holding time) which is exponentially distributed with mean $1 / \mu$.

The system parameters, chosen for vehicular MT's traveling through a city center, are as follows: $300 \mathrm{~m} \leq R \leq$ $1000 \mathrm{~m}, T_{m_{-} \max }=40 \mathrm{~s}, T_{s_{-} \max }=40 \mathrm{~s}, V_{\max }=$ $70 \mathrm{~km} / \mathrm{h}, 1 / \mu=120 \mathrm{~s}$, and $\phi=100^{\circ}$. The range of $R$ is about the size of microcells which can handle the vehicular traffic. $T_{m_{-} \max }$ is about the time required for an MT to travel through two city blocks, and $T_{s_{-} \max }$ is about the time interval of a red traffic light in a city. $V_{\max }$ is approximately the maximum speed of an MT traveling in a city. The $1 / \mu$ value reflects the mean call holding time for cellular calls in busy hours. The $\phi$ value reflects the fact that vehicular MT's are unlikely to reverse direction on the road. Different traffic conditions are simulated with $p=0.0$ for light traffic, $p=0.25$ for moderate traffic, and $p=0.5$ for heavy traffic, with $R$ ranging from 300 to $1000 \mathrm{~m}$. Let $\alpha, \beta$, and $\gamma$ be the ratios between the number of MT's originating calls from

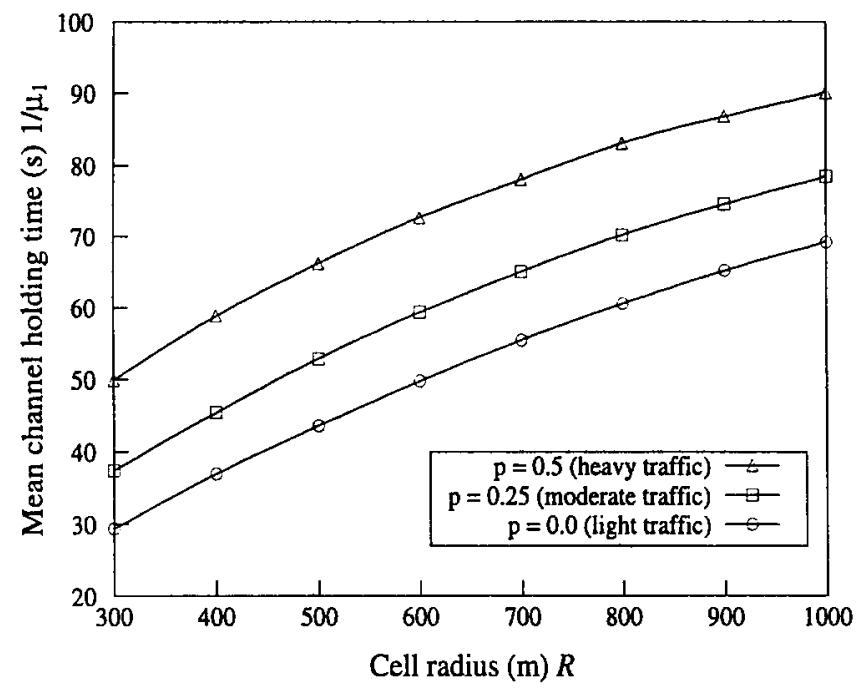

Fig. 5. Mean channel holding time versus cell size under different traffic conditions.

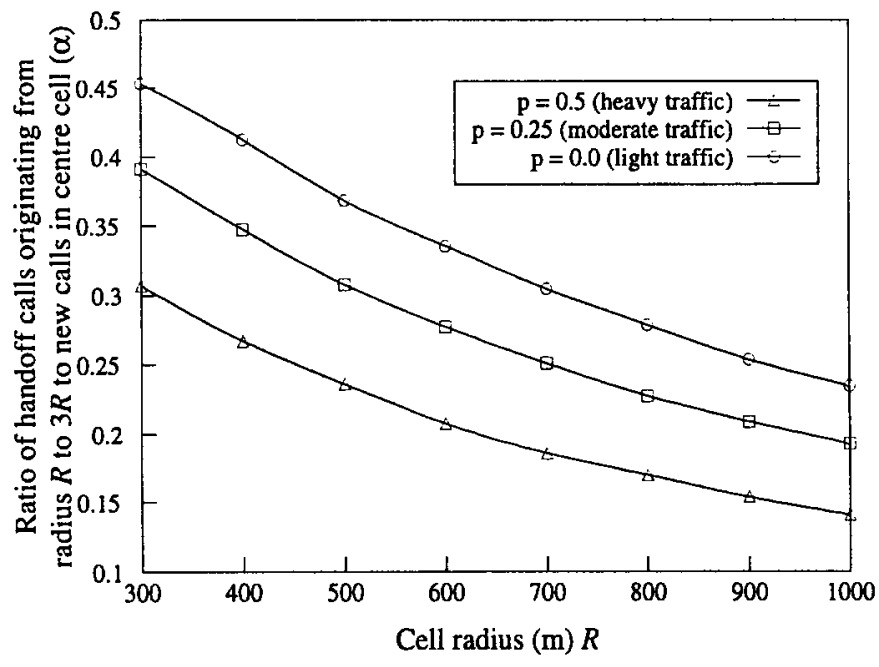

Fig. 6. Values of $\alpha$ versus cell size under different traffic conditions.

$R<r<3 R, 3 R \leq r<5 R$, and $5 R \leq r<7 R$, respectively, and moving into the center cell before call termination, to the number of new calls originated in the center cell, over a given time interval. The values of $\alpha, \beta$, and $\gamma$ correspond to the ratios of handoff calls to new calls in the center cell, where the handoff calls originate from the respective layers of cells 0,1 , and 2 cell diameters away from the center cell.

Simulation results in Fig. 5 show that the mean channel holding time increases with cell size and road traffic. In addition, the results show that the channel holding time probability density function is approximately negative exponential, with the approximation getting closer for larger cells. Figs. 6-8 show the effects of cell size and road traffic condition on the values of $\alpha, \beta$, and $\gamma$, respectively. Fig. 8 shows that a relatively small number of MT's originating calls from $5 R \leq r<7 R$ eventually goes into the center cell before call termination, especially when $R>500 \mathrm{~m}$. In comparison, the values of $\alpha$ are much greater than the corresponding values of $\beta$ and $\gamma$, showing that most handoff calls originate from 


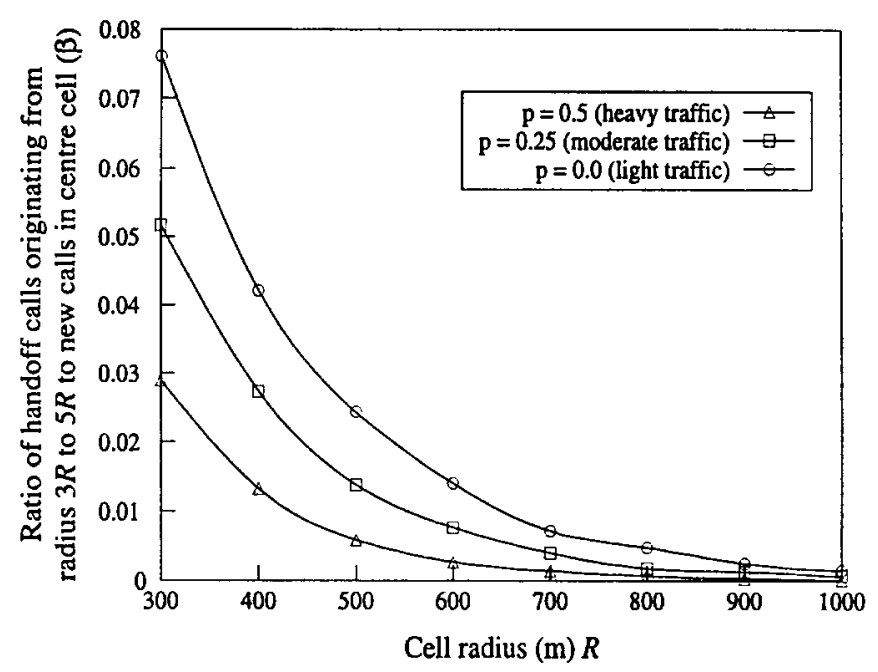

Fig. 7. Values of $\beta$ versus cell size under different traffic conditions.

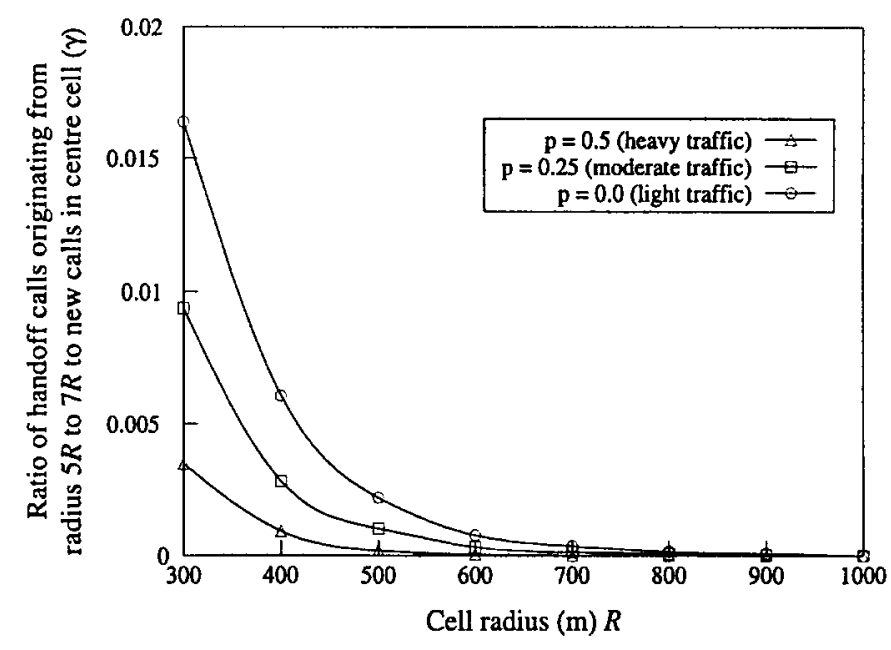

Fig. 8. Values of $\gamma$ versus cell size under different traffic conditions.

the immediate neighboring cells. The results for $R=500 \mathrm{~m}$ and $p=0.25$ (moderate traffic) are chosen for evaluation of the dimensions of the BS-MSC and MSC-MSC links for the respective network configuration in the next section.

Note that our mobility model does not take into account propagation effects. Consequently, the handoff modeling and subsequent analysis and results do not account for such phenomena as "rescue handoffs" necessitated by sudden degradations of link quality within a cell.

\section{Dimensioning BS-MSC AND MSC-MSC LINKS}

In the CDMA digital cellular system developed by Qualcomm, there are a maximum of 62 uplink traffic channels and 63 downlink traffic channels, including synchronization and paging channels which are convertible to traffic channels, available at each BS [4]. For simplicity, we assume a maximum of 62 uplink/downlink traffic channels in each BS in this paper. The link capacities of the proposed network configurations required to support the maximum possible traffic level in each cell are analyzed, assuming uniform distribution of MT's in all cells. Nonuniform user distribution may result in an increase or decrease of intercluster handoffs, necessitating appropriate adjustments of our results. The analysis assumes that blocked new calls are dropped and lost from the system, such that redials are considered new and independent arrivals. This assumption is a common practice in traffic engineering [20].

\section{A. Link Capacity for Configuration I}

The objective is to determine the number of channels required for direct link $L_{A 1}$ and cross link $L_{A 2}$ in Fig. 1. By symmetry, links $L_{B 2}$ and $L_{B 1}$ require the same numbers of channels as links $L_{A 1}$ and $L_{A 2}$, respectively. Let $\lambda_{o}$ be the new call arrival rate in a microcell. Assume that all arrival processes are Poisson. From the results of Section II, for $R=500 \mathrm{~m}$ and $p=0.25$, the channel holding time for direct link $L_{A 1}$ in a microcell is exponentially distributed with mean $1 / \mu_{1}=53$ s, and $\alpha=0.308, \beta=0.0138$, and $\gamma=0.001$. Because the values of $\beta$ and $\gamma$ are relatively small compared to the value of $(1+\alpha)$, the factors, $\beta$ and $\gamma$ due to handoffs from calls originating from more than 1 cell away are neglected. The total call arrival rate at direct link $L_{A 1}$ and crosslink $L_{A 2}$ are therefore $(1+\alpha) \lambda_{o}$ and $(2 / 3) \alpha \lambda_{o}$, respectively. For crosslink $L_{A 2}$, assume that the channel holding time for the MT's passing through the handoff region is exponentially distributed with mean $1 / \mu_{2}$. From [21], for $R=500 \mathrm{~m}$, the channel holding time for an MT passing through the handoff region is between $1 \mathrm{~s}$ and $18 \mathrm{~s}$, assuming that the MT moves along the straight line connecting the two BS's. Let $1 / \mu_{2}=15 \mathrm{~s}$.

There are two channel assignment schemes resulting in different holding times over the crosslinks during soft handoffs. The two assignment schemes are described as follows.

1) Channel Assignment Scheme A: In channel assignment scheme $A$, when an MT hands off from cell 1 to 2 , the handoff is successful when there is a channel available in direct link $L_{B 2}$; otherwise, the call is dropped immediately. The blocking probability $P_{B}$ for each link is given by the Erlang B formula [22]

$$
P_{B}=\frac{\rho^{m} / m !}{\sum_{n=0}^{m} \rho^{n} / n !}
$$

where $\rho=\lambda / \mu$ is the Erlang load and $m$ is the number of channels in the corresponding link. Let $P_{B}\left(L_{A 2}\right)$ and $P_{B}\left(L_{B 2}\right)$ be the blocking probabilities of crosslink $L_{A 2}$ and direct link $L_{B 2}$, respectively. The handoff blocking probability $P_{B h 1 A}$ between the two cell clusters is given by

$$
P_{B h 1 A}=P_{B}\left(L_{A 2}\right)+\left[1-P_{B}\left(L_{A 2}\right)\right] P_{B}\left(L_{B 2}\right) .
$$

Subject to the constraint that the total number of channels in links $L_{A 2}$ and $L_{B 2}$ equals 62 , the cell-cluster handoff blocking probability $P_{B h 1 A}$ can be minimized with respect to the dimensions of these links. For $\lambda_{o}=2200 / \mathrm{h} /$ cell, Fig. 9 shows that 55 channels should be assigned to direct link $L_{B 2}$ and the remaining seven channels assigned to crosslink $L_{A 2}$ to minimize $P_{B h 1 A}$. Table I shows the traffic parameters for scheme $A$ in Configuration $\mathrm{I}$ and the channel dimensions minimizing $P_{B h 1 A}$ at $2000 \leq \lambda_{o} \leq 2500 / \mathrm{h} /$ cell. Fig. 10 
TABLE I

TRAFFic PARAmeters For ChanNel Assignment Scheme $A$ of Configuration I

\begin{tabular}{c|c|c|c|c}
\hline \multirow{2}{*}{$\begin{array}{c}\text { Connection links } \\
\text { of configuration I }\end{array}$} & \multirow{2}{*}{ Arrival rate } & \multirow{2}{*}{$\begin{array}{c}\text { Channel } \\
\text { bolding time }\end{array}$} & \multicolumn{2}{|c}{$\begin{array}{c}\text { Channel dimensions to minimize } \\
P_{\text {BhIA }}\end{array}$} \\
\cline { 4 - 5 } & & & $\lambda_{0}=2000$ & $2100 \leq \lambda_{0} \leq 2500$ \\
\hline $\mathrm{L}_{\mathrm{A} 1} \cdot \mathrm{L}_{\mathrm{B} 2}$ & $(1+0) \lambda_{0}$ & $1 / \mu_{1}$ & 54 & 55 \\
\hline $\mathrm{L}_{\mathrm{A} 2} \cdot \mathrm{L}_{\mathrm{B} 1}$ & $(2 / 3) 0 \lambda_{o}$ & $1 / \mu_{2}$ & 8 & 7 \\
\hline
\end{tabular}

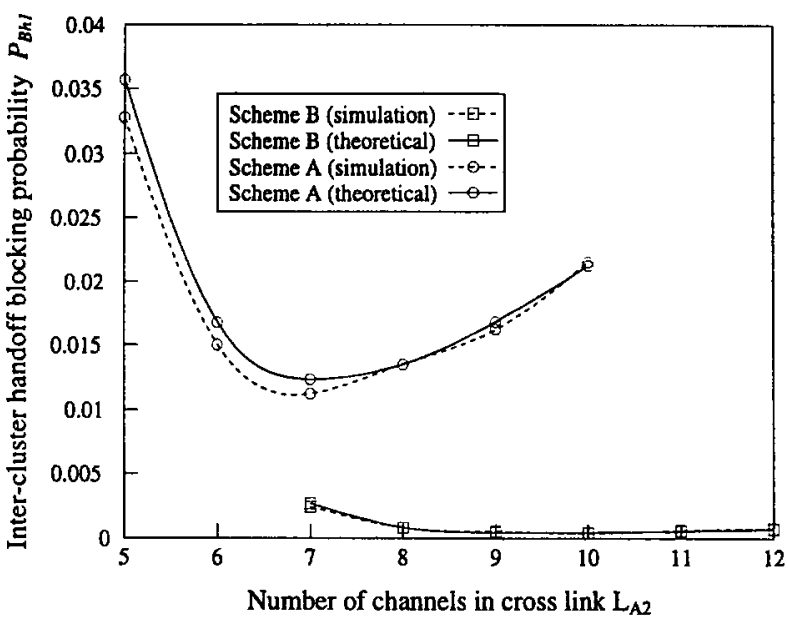

Fig. 9. Intercluster handoff blocking probability in Configuration I, $P_{B h 1}$, versus number of channels in $L_{A 2}$ at $\lambda_{o}=2200 / \mathrm{h} / \mathrm{cell}$.

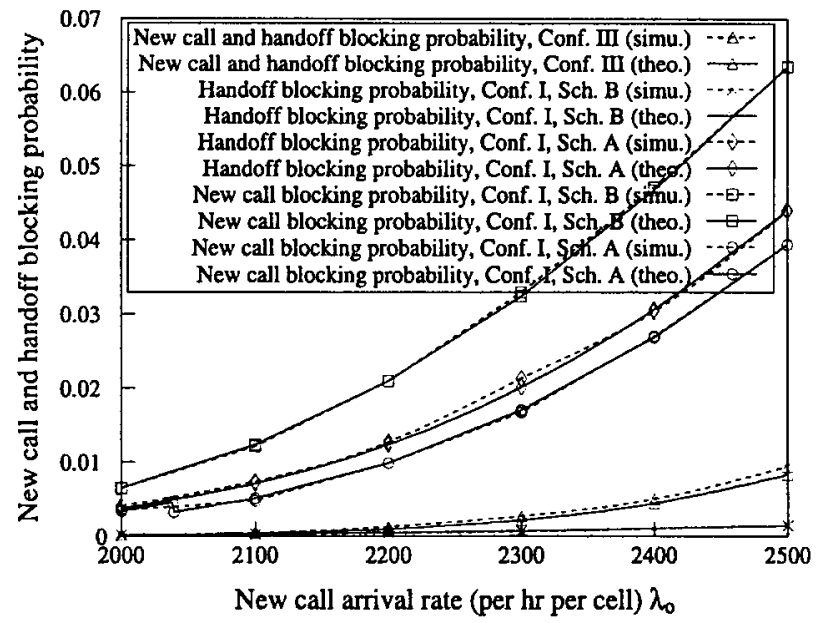

Fig. 10. New call and handoff blocking probability with optimal channel dimensions in Configurations I and III.

shows the new call blocking probability and handoff blocking probability $P_{B h 1 A}$ with optimal channel dimensions at $2000 \leq$ $\lambda_{0} \leq 2500 / \mathrm{h} /$ cell, respectively. In this case, the blocking probability in direct link $L_{B 2}$ is taken as the new call blocking probability $P_{B n}$.

Since there are 62 uplink/downlink channels, this number is also the minimum for the total number of channels in the direct link and crosslink. In fact, we can reduce the blocking probabilities by increasing the total number of direct link and crosslink channels to more than 62. Fig. 11 shows the optimal dimensions of the crosslinks at $\lambda_{o}=2200 / \mathrm{h} / \mathrm{cell}$, when there are more than 62 channels in total between the

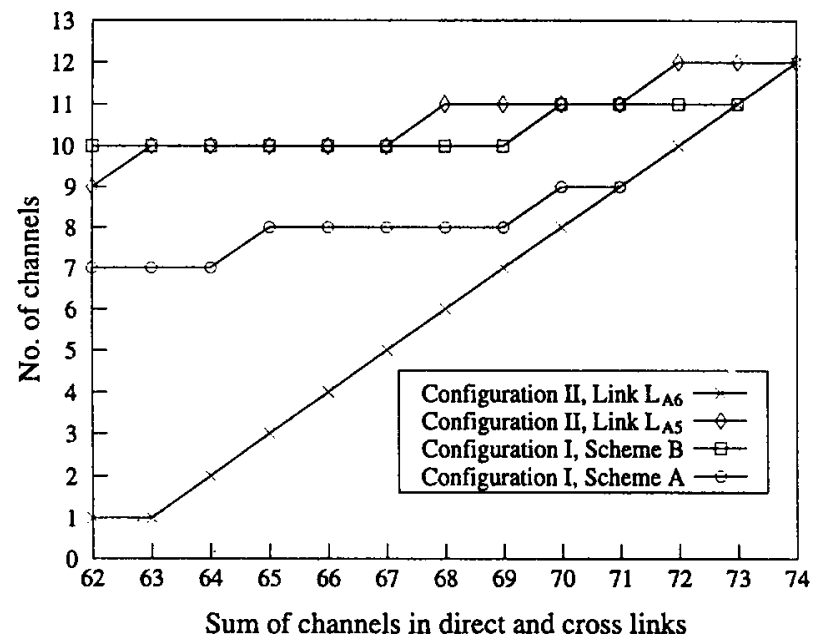

Fig. 11. Channel dimensions for direct and crosslinks at $\lambda_{o}=2200 / \mathrm{h} / \mathrm{cell}$ in Configuration I and at $\lambda_{o}=2300 / \mathrm{h} / \mathrm{cell}$ in Configuration II.

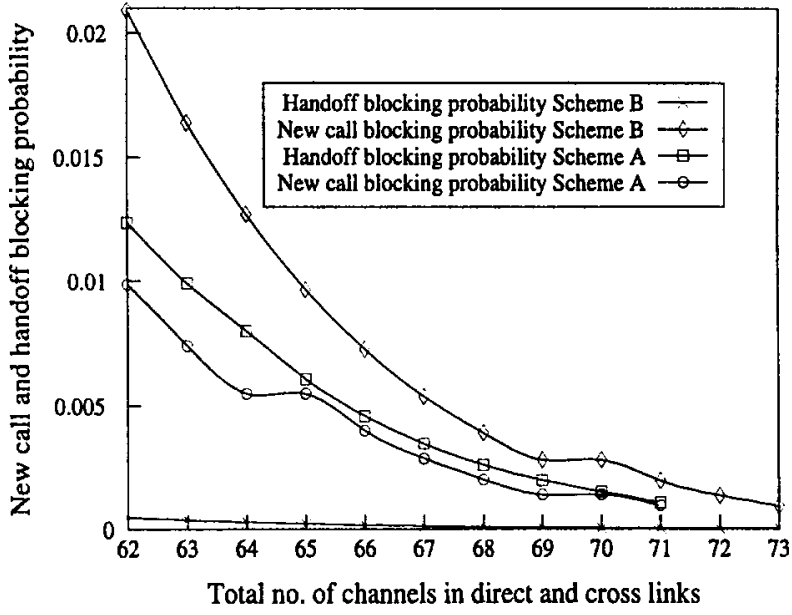

Fig. 12. New call and handoff blocking probability versus total number of channels in direct and crosslinks at $\lambda_{o}=2200 / \mathrm{h} /$ cell in Configuration I.

direct and crosslinks. Fig. 12 shows that by adding extra channels above the sum total of 62 channels between the direct and crosslinks, the new call and handoff blocking probabilities are significantly reduced. The decrease of new call blocking probability is not monotonic relative to the total number of channels; the steps occur where the optimal channel partition adds one more channel to the crosslink, as indicated by Fig. 11, leaving the number of channels in the direct link unchanged.

According to the mobility model in Section III, channel holding time $1 / \mu_{1}$ varies between 30 and $90 \mathrm{~s}$ and the value of $\alpha$ ranges from 0.15 to 0.45 . The optimal channel dimensions to minimize handoff blocking probability $P_{B h 1 A}$ for these situations have also been investigated, and the results are presented in Table II for different $1 / \mu_{1}$ and $\alpha$ with $P_{B n}<0.01$. In general, the optimal number of crosslinks $L_{A 2}$ increases as $\alpha$ and as $1 / \mu_{1}$ decreases. Fig. 13 shows the maximum new call arrival rate $\lambda_{o}$ under these situations. The handoff blocking probabilities $P_{B h 1 A}$ in all these cases are between 0.01 and 0.014 .

2) Channel Assignment Scheme B: In channel assignment scheme $B$, if there is no channel available in direct link $L_{B 2}$ 
TABLE II

Optimal Channel Dimensions Versus Channel Holding Time and $\alpha$ with $P_{B n}<0.01$ for Scheme $A$ in Configuration I

\begin{tabular}{c|c|c|c|c|c|c|c|c}
\hline \multirow{2}{*}{$\mathrm{L}_{\mathrm{A} 1}, \mathrm{~L}_{\mathrm{A} 2}$} & \multicolumn{7}{c|}{ Channel holding time $1 / \mu_{1}(\mathrm{~s})$} \\
\cline { 2 - 9 } & 30 & 40 & 50 & 60 & 70 & 80 & 90 \\
\hline \multirow{4}{*}{0.15} & 55,7 & 56,6 & 56,6 & 57,5 & 57,5 & 57,5 & 57,5 \\
\cline { 2 - 9 } & 0.20 & 54,8 & 55,7 & 56,6 & 56,6 & 56,6 & 57,5 & 57,5 \\
\cline { 2 - 9 } & 0.25 & 53,9 & 54,8 & 55,7 & 56,6 & 56,6 & 56,6 & 57,5 \\
\cline { 2 - 9 } & 0.30 & 53,9 & 54,8 & 55,7 & 55,7 & 56,6 & 56,6 & 56,6 \\
\cline { 2 - 9 } & 0.35 & 52,10 & 53,9 & 54,8 & 55,7 & 55,7 & 56,6 & 56,6 \\
\hline & 0.40 & 52,10 & 53,9 & 54,8 & 55,7 & 55,7 & 55,7 & 56,6 \\
\cline { 2 - 9 } & 0.45 & 51,11 & 53,9 & 54,8 & 55,7 & 55,7 & 55,7 & 56,6 \\
\hline
\end{tabular}

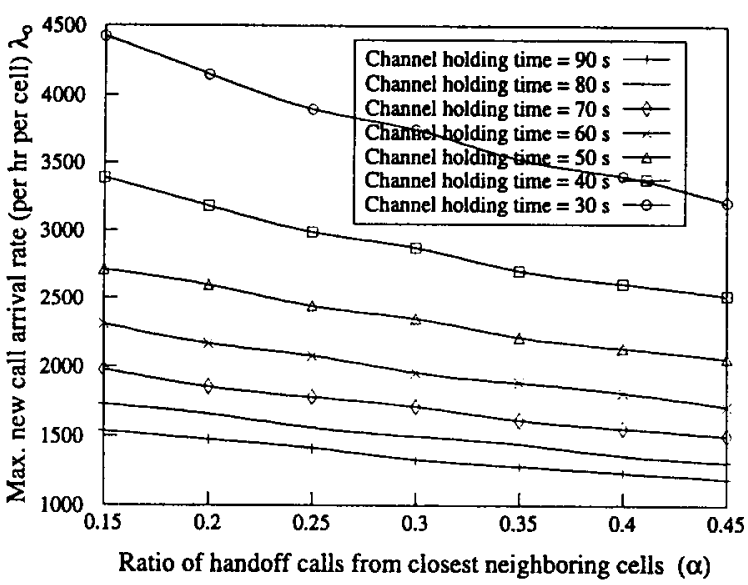

Fig. 13. Maximum new call arrival rate with $P_{B n}<0.01$ versus $\alpha$ and different channel holding times for optimal channel dimensions of scheme $A$ in Configuration I.

when an MT hands off from cell 1 to 2 , the call is maintained by the existing channel in crosslink $L_{A 2}$ until either the MT leaves cell 2 or a channel in $L_{B 2}$ is available. If the MT moves away from cell 2 into another cell in cluster $B$ while the call is still handled by $L_{A 2}$, the call will be dropped. This scheme helps to improve (reduce) the handoff blocking probability between cell clusters.

The derivation of the channel dimensions in scheme $B$ is similar to the derivation for priority scheme II in [13] without any guard channel, except for the finite queue size in our case. Assume that there are $C$ and $C_{q}$ channels in direct link $L_{A 1}$ and crosslink $L_{A 2}$, respectively. By symmetry, links $L_{B 2}$ and $L_{B 1}$ require the same number of channels as links $L_{A 1}$ and $L_{A 2}$, respectively. A new call in cell 2 is blocked when all $C$ channels in direct link $L_{B 2}$ are fully occupied. At this moment, if there is a handoff call from crosslink $L_{A 2}$, the call will be maintained by $L_{A 2}$ as long as the MT is in cell 2 . This is equivalent to putting the handoff call in a queue waiting for a channel in $L_{B 2}$ to be available. When a channel becomes available in $L_{B 2}$, the channel will be first assigned to a handoff call waiting in the queue in crosslink $L_{A 2}$. If there is more than one handoff call waiting in the queue in $L_{A 2}$, the first-come, first-served (FCFS) queueing discipline is used. That is, the handoff calls in $L_{A 2}$ are allowed to queue with a buffer size close to the number of channels available in $L_{A 2}$. The buffer size can be less than the number of channels in $L_{A 2}$ because $L_{A 2}$ serves the handoff calls both from cell 2 to 1 and from cell 1 to 2 . However, for simplicity, we assume that the buffer size equals the number of channels $C_{q}$ in crosslink $L_{A 2}$.

When all of the channels in direct link $L_{B 2}$ are fully occupied, the waiting time for the handoff calls in crosslink $L_{A 2}$ depends on the remaining service time of the MT's in cell 2 , which is exponentially distributed with mean $1 / \mu_{1}$. Let $X$ be the random variable representing the time from the instant a handoff call joins the queue in $L_{A 2}$ to the first instant that a channel is released in $L_{B 2}$. Because there are $C$ channels in $L_{B 2}$ with service time $1 / \mu_{1}$ each, $X$ is exponentially distributed with mean $1 /\left(C \mu_{1}\right)$.

Let $T_{i}$ be the remaining time for the handoff call in the $i$ th queue position before the MT moves out of cell 2 . Under the memoryless property, the random variable $T_{i}$ is the same as the channel holding time random variable which is exponentially distributed with mean $1 / \mu_{1}$. Let $E$ be the state or occupancy of the queue. From the description of this channel assignment scheme and the properties of the exponential distribution, the following equation is obtained:

$$
\begin{aligned}
\operatorname{Pr}\{E(t+h) & =C+k-1 \mid E(t)=C+k\} \\
& =\operatorname{Pr}\left\{X \leq h \text { or } T_{1} \leq h \text { or } \cdots T_{k} \leq h\right\} \\
& =1-\operatorname{Pr}\{X>h\} \operatorname{Pr}\left\{T_{1}>h\right\} \cdots \operatorname{Pr}\left\{T_{k}>h\right\} \\
& =1-e^{-(C+k) \mu_{1} h} .
\end{aligned}
$$

From (5), it follows that state transitions are given by the birth-death process, and may be represented by a Markov chain [22], from which the state probabilities $P_{n}$ can be easily found

$$
\begin{gathered}
P_{n}= \begin{cases}\frac{(1+\alpha) \lambda_{o}}{n \mu_{1}} P_{n-1}, & n=1,2, \cdots, C \\
\frac{\frac{1}{3} \alpha \lambda_{o}}{n \mu_{1}} P_{n-1}, & n=C+1, \cdots, C+C_{q}\end{cases} \\
\sum_{n=0}^{C+C_{q}} P_{n}=1 .
\end{gathered}
$$

Solving (6) and (7), the following results are obtained:

$$
\begin{gathered}
P_{0}=\left\{\sum_{n=0}^{C}\left[\frac{(1+\alpha) \lambda_{o}}{\mu_{1}}\right]^{n} \frac{1}{n !}+\sum_{n=C+1}^{C+C_{q}}\left[\frac{(1+\alpha) \lambda_{o}}{\mu_{1}}\right]^{C}\right. \\
\left.\cdot\left[\frac{\frac{1}{3} \alpha \lambda_{o}}{\mu_{1}}\right] \frac{1}{n !}\right\} \\
P_{n}=\left\{\begin{array}{l}
{\left[\frac{(1+\alpha) \lambda_{o}}{\mu_{1}}\right]^{n} \frac{1}{n !} P_{0},} \\
{\left[\frac{(1+\alpha) \lambda_{o}}{\mu_{1}}\right]^{C}\left[\frac{\frac{1}{3} \alpha \lambda_{o}}{\mu_{1}}\right]^{n-C} \frac{1}{n !} P_{0},}
\end{array}\right.
\end{gathered}
$$

The blocking probability $P_{B o}$ for an originating call arriving at direct link $L_{B 2}$ is given by

$$
P_{B o}=\sum_{n=C}^{C+C_{q}} P_{n} .
$$


The average queue length $N_{Q}$ for the handoff calls is obtained by the following equation:

$$
N_{Q}=\frac{\sum_{n=0}^{C_{q}} n P_{C+n}}{P_{B o}} .
$$

By Little's theorem [22], the average waiting time $w$ in the queue is given by

$$
w=\frac{N_{Q}}{\frac{1}{3} \alpha \lambda_{o}} .
$$

Let $P_{f h}$ be the probability of a handoff attempt failure due to an MT in the queue leaving cell 2 before it gets a channel in $L_{B 2}$. Let $P_{f h \mid k}$ be the probability that the handoff attempt fails given that the MT enters position $k+1$ in the queue. $P_{f h}$ can be expressed in terms of state probability $P_{n}$ and $P_{f h \mid k}$

$$
P_{f h}=\sum_{k=0}^{C_{q}-1} P_{C+k} P_{f h \mid k}
$$

where $P_{f h \mid k}$ is given by the following equation [13]:

$$
P_{f h \mid k}=1-\left(\frac{C}{C+1}\right) \prod_{i=1}^{k}\left[1-\left(\frac{1}{C+1}\right)\left(\frac{1}{2}\right)^{i}\right] .
$$

The blocking probability $P_{h}\left(L_{B 2}\right)$ that a handoff call is blocked in direct link $L_{B 2}$ is given by

$$
P_{h}\left(L_{B 2}\right)=P_{f h}+P_{C+C_{q}} .
$$

The weighted blocking probability $P_{B}\left(L_{B 2}\right)$ of direct link $L_{B 2}$ for all calls is as follows:

$$
P_{B}\left(L_{B 2}\right)=\frac{\left(\lambda_{o}+\left(1-\frac{1}{3}\right) \alpha \lambda_{\circ}\right) P_{B o}+\left(\frac{1}{3} \alpha \lambda_{h}\right) P_{h}\left(L_{B 2}\right)}{\lambda_{o}+\lambda_{h}} .
$$

For crosslink $L_{A 2}$, the probability that $L_{A 2}$ is blocked given that $L_{B 2}$ is not blocked is given by (3) with arrival rate $=(2 / 3) \alpha \lambda_{o}$, mean service time $=1 / \mu_{2}$, and $m=C_{q}$. The probability that $L_{A 2}$ is blocked given that $L_{B 2}$ is blocked is given by the equation of a two-dimensional (2-D) Markov chain [22]:

$$
\operatorname{Pr}\left\{L_{A 2} \text { blocked } \mid L_{B 2} \text { blocked }\right\}=\frac{\sum_{n=0}^{C_{q}} \frac{\rho_{1}^{n} \rho_{2}^{C_{q}-n}}{n !\left(C_{q}-n\right) !}}{\sum_{i=0}^{C_{q}} \sum_{j=0}^{C_{q}-i} \frac{\rho_{1}^{i} \rho_{2}^{j}}{i ! j !}}
$$

where $\rho_{1}=(1 / 3) \alpha \lambda_{\circ} / \mu_{2}$ and $\rho_{2}=(1 / 3) \alpha \lambda_{\circ}\left(1 / \mu_{2}+w\right)$. The mean service time in the Erlang load $\rho_{2}$ is simply the sum of two mean service times $1 / \mu_{2}$ and $w$. The blocking probability $P_{B}\left(L_{A 2}\right)$ for crosslink $L_{A 2}$ is as follows:

$$
\begin{aligned}
P_{B}\left(L_{A 2}\right)= & \operatorname{Pr}\left\{L_{A 2} \text { blocked } \mid L_{B 2} \text { blocked }\right\} \cdot P_{B o} \\
& +\operatorname{Pr}\left\{L_{A 2} \text { blocked } \mid L_{B 2} \text { not blocked }\right\} \\
& \cdot\left(1-P_{B o}\right) .
\end{aligned}
$$

TABLE III

Traffic Parameters For Channel Assignment Scheme $B$ of Configuration I

\begin{tabular}{c|c|c|c}
\hline \multirow{2}{*}{$\begin{array}{c}\text { Connection links of } \\
\text { configuration I }\end{array}$} & Arrival rate & \multirow{2}{*}{$\begin{array}{c}\text { Channel holding } \\
\text { time }\end{array}$} & $\begin{array}{c}\text { Channel dimensions to } \\
\text { minimize } P_{B h l B}\end{array}$ \\
\cline { 3 - 3 } & & $1 / \mu_{1}$ & $2000 \leq \lambda_{o} \leq 2500$ \\
\hline $\mathrm{L}_{\mathrm{A} 1}, \mathrm{~L}_{\mathrm{B} 2}$ & $(1+\alpha) \lambda_{o}$ & $1 / \mu_{2}$ & 52 \\
\hline $\begin{array}{c}\mathrm{L}_{\mathrm{A} 2}, \mathrm{~L}_{\mathrm{B} 1} \text { given } \\
\mathrm{L}_{\mathrm{B} 2}, \mathrm{~L}_{\mathrm{A} 1} \text { not blocked }\end{array}$ & $(2 / 3) \alpha \lambda_{o}$ & \multirow{2}{*}{10} \\
\hline $\begin{array}{c}\mathrm{L}_{\mathrm{A} 2}, \mathrm{~L}_{\mathrm{B} 1} \text { given } \\
\mathrm{L}_{\mathrm{B} 2}, \mathrm{~L}_{\mathrm{A} 1} \text { blocked }\end{array}$ & $(1 / 3) \alpha \lambda_{o}$ & $1 / \mu_{2}$ & \\
\cline { 2 - 3 } & $(1 / 3) \alpha \lambda_{o}$ & $1 / \mu_{2}+\omega$ & \\
\hline
\end{tabular}

The blocking probability $P_{B h 1 B}$ for handoffs between the two cell clusters is given by

$$
P_{B h 1 B}=P_{B}\left(L_{A 2}\right)+\left[1-P_{B}\left(L_{A 2}\right)\right] \cdot P_{f h} .
$$

Again, subject to the constraint that the total number of channels in $L_{A 2}$ and $L_{B 2}$ equals 62 , the cell-cluster handoff blocking probability $P_{B h 1 B}$ can be minimized with respect to the dimensions of links $L_{A 2}$ and $L_{B 2}$. For $\lambda_{o}=2200 / \mathrm{h} / \mathrm{cell}$, Fig. 9 shows that 52 channels should be assigned to direct link $L_{B 2}$ and the remaining ten channels to crosslink $L_{A 2}$ in order to minimize $P_{B h 1 B}$. However, note that $P_{B h 1 B}$ is much less sensitive to the channel partition compared to $P_{B h 1 A}$. Table III shows the traffic parameters for scheme $B$ in Configuration I and the channel dimensions minimizing $P_{B h 1 B}$ at $2000 \leq$ $\lambda_{o} \leq 2500 / \mathrm{h} /$ cell. The blocking probability $P_{B}\left(L_{A 2}\right)$ in crosslink $L_{A 2}$ is very low. For example, it is less than 0.00007 for $\lambda_{o}=2500 / \mathrm{h} /$ cell. The blocking probability in direct link $L_{B 2}$ can be taken as the new call blocking probability. Fig. 10 shows the new call blocking probability $P_{B n}$ and handoff blocking probability $P_{B h 1 B}$ with optimal channel dimensions at $2000 \leq \lambda_{o} \leq 2500 / \mathrm{h} /$ cell, respectively. From Figs. 9 and 10 , the handoff blocking probability $P_{B h 1 B}$ in scheme $B$ is much lower than the handoff blocking probability $P_{B h 1 A}$ in scheme $A$; however, the new call blocking probability $P_{B n}$ in scheme $B$ is higher than that in scheme $A$. These results show that scheme $B$ enables new call blocking performance to be traded off for better handoff call blocking performance. The average waiting time $w$ in the queue of crosslink $L_{A 2}$ is $1 \mathrm{~s}$.

Similar to the consideration in scheme $A$, more than 62 total channels can be assigned to the direct and crosslinks to reduce the blocking probabilities. Fig. 11 shows the optimal dimensions of the crosslinks when there is more than a total of 62 channels to be partitioned between the direct and crosslinks, and Fig. 12 shows that the extra channels significantly reduce the new call blocking probability. Reduction of handoff blocking probability is not as pronounced as this probability is already very low.

As in scheme $A$, the optimal channel dimensions to minimize handoff blocking probability $P_{B h 1 B}$ for various channel holding times $1 / \mu_{1}$ and $\alpha$ have also been investigated. The optimal channel dimensions in Table IV with $P_{B n}<0.01$ show the same trends with respect to $1 / \mu_{1}$ and $\alpha$ as in Table II. Fig. 14 shows the maximum new call arrival rate $\lambda_{o}$ which can be attained under these situations. In comparison with Fig. 13, the maximum attainable new call arrival rate is 
TABLE IV

Optimal Channel Dimensions Versus Channel Holding Time AND $\alpha$ With $P_{B n}<0.01$ For Scheme $B$ in Configuration I

\begin{tabular}{c|c|c|c|c|c|c|c|c}
\hline \multirow{2}{*}{$\mathrm{L}_{\mathrm{A} 1}, \mathrm{~L}_{\mathrm{A} 2}$} & \multicolumn{7}{|c|}{ Channel holding time $1 / \mu,(\mathrm{s})$} \\
\cline { 2 - 10 } & 30 & 40 & 50 & 60 & 70 & 80 & 90 \\
\hline \multirow{4}{*}{0.15} & 53,9 & 54,8 & 54,8 & 55,7 & 55,7 & 55,7 & 56,6 \\
\cline { 2 - 10 } & 0.20 & 52,10 & 53,9 & 53,9 & 54,8 & 54,8 & 55,7 & 55,7 \\
\cline { 2 - 10 } & 0.25 & 51,11 & 52,10 & 53,9 & 53,9 & 54,8 & 54,8 & 55,7 \\
\cline { 2 - 10 } & 0.30 & 50,12 & 51,11 & 52,10 & 53,9 & 53,9 & 54,8 & 54,8 \\
\cline { 2 - 10 } & 0.35 & 49,13 & 51,11 & 52,10 & 53,9 & 53,9 & 54,8 & 54,8 \\
\cline { 2 - 10 } & 0.40 & 49,13 & 50,12 & 51,11 & 52,10 & 53,9 & 53,9 & 54,8 \\
\cline { 2 - 9 } & 0.45 & 48,14 & 50,12 & 51,11 & 52,10 & 52,10 & 53,9 & 53,9 \\
\hline
\end{tabular}

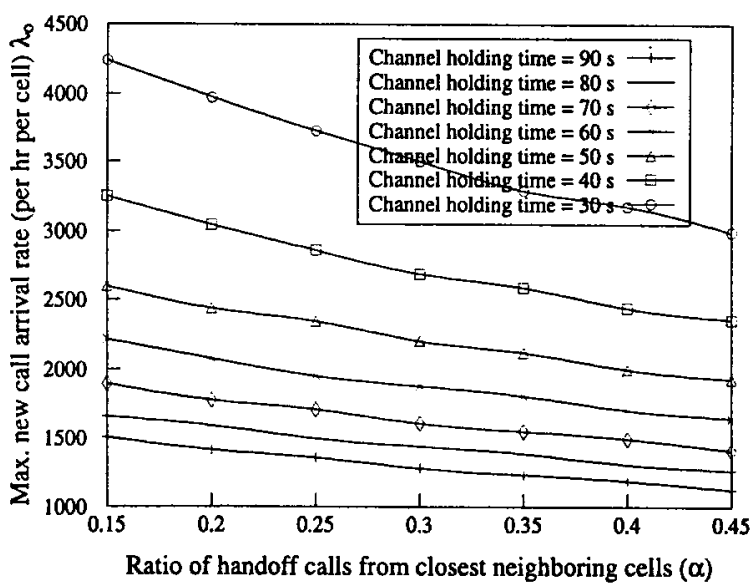

Fig. 14. Maximum new call arrival rate with $P_{B n}<0.01$ versus $\alpha$ and different channel holding times for optimal channel dimensions of scheme $B$ in Configuration I.

slightly lower for channel assignment scheme $B$. The handoff blocking probabilities $P_{B h 1 B}$ between the cell clusters in all of these cases are between 0.00019 and 0.0003 . These are significantly reduced compared to the corresponding values of $P_{B h 1 A}$ for channel assignment scheme $A$.

\section{B. Link Capacity for Configuration II}

As shown in Fig. 2, appropriate numbers of channels needs to be assigned to links $L_{\mathrm{Ak}}$ for $k=3,4,5$, and 6 . By symmetry, links $L_{\mathrm{Bk}}$ require the same capacity as links $L_{\mathrm{A}(9-\mathrm{k})}$ for $k=3,4,5$, and 6 . The traffic going from cell 3 to 4 and then back to 3 is neglected in accordance with the assumptions of the mobility model. The number of channels required for links $L_{\mathrm{A} 3}, L_{\mathrm{A} 5}$, and $L_{\mathrm{A} 6}$ are governed by (3). Link $L_{\mathrm{A} 4}$ is utilized by new traffic with rate $\lambda_{o}$ originating from cell 4 , traffic with rate $(2 / 3) \alpha \lambda_{o}$ due to handoffs from surrounding microcells in cell cluster $A$, as well as intercluster handoff traffic with rate $(1 / 3)(\beta+\gamma) \lambda_{o}$ originating from cell cluster $B$. Since $(1 / 3)(\beta+\gamma) \ll 1+(2 / 3) \alpha$, the last type of traffic can be ignored. Thus, the number of channels required for link $L_{\mathrm{A} 4}$ is still governed by (3). Let $P_{B}\left(L_{A k}\right)$ and $P_{B}\left(L_{B k}\right)$ be the blocking probabilities of links $L_{\mathrm{Ak}}$ and $L_{\mathrm{Bk}}$ for $k=3,4,5$, and 6 , respectively. The overall handoff blocking probability $P_{B h 2}$ between cell clusters for Configuration II is estimated from the weighted average of handoff success probabilities for traffic over cell cluster $A$ in Fig. 2:

$$
\begin{aligned}
P_{B h 2}=1-\{ & \alpha \lambda_{o}\left[1-P_{B}\left(L_{A 3}\right)\right]+\frac{4}{6} \alpha \lambda_{o}\left[1-P_{B}\left(L_{A 4}\right)\right] \\
& +\frac{2}{6} \alpha \lambda_{\circ}\left[1-P_{B}\left(L_{A 5}\right)\right] \\
& +\frac{4}{6} \beta \lambda_{o}\left[1-P_{B}\left(L_{A 3}\right)\right]\left[1-P_{B}\left(L_{A 4}\right)\right] \\
& +\frac{2}{6} \beta \lambda_{\circ}\left[1-P_{B}\left(L_{A 4}\right)\right]\left[1-P_{B}\left(L_{A 5}\right)\right] \\
& +\frac{2}{6} \beta \lambda_{\circ}\left[1-P_{B}\left(L_{A 3}\right)\right] \\
& \cdot\left[1-P_{B}\left(L_{A 5}\right)\right]\left[1-P_{B}\left(L_{A 6}\right)\right] \\
& +\frac{2}{6} \gamma \lambda_{o}\left[1-P_{B}\left(L_{A 3}\right)\right]\left[1-P_{B}\left(L_{A 4}\right)\right] \\
& \left.\cdot\left[1-P_{B}\left(L_{A 5}\right)\right]\left[1-P_{B}\left(L_{A 6}\right)\right]\right\} / \\
& \cdot\left\{\left[2 \alpha+\frac{4}{3} \beta+\frac{2}{6} \gamma\right] \lambda_{\circ}\right\} .
\end{aligned}
$$

In the numerator on the right-hand side of the difference in (20), the first three terms account for handoff successes in cells 3,4 , and 5 , respectively, due to new calls from immediate neighboring cells in cluster $A$, the next three terms account for handoff successes in cells 4, 5, and 6, respectively, due to new calls from 1 cell removed in cluster $A$, and the final term accounts for handoff successes in cell 6 due to new calls from 2 cells removed in cluster $A$. The extra $\left[1-P_{B}\left(L_{A 3}\right)\right]$ in the last two terms accounts for handoffs into $L_{\mathrm{B} 6}$ in cell 6. An intercluster handoff from cluster $A$ to $B$ is successful only if the call is handed off successfully through cells 3 , 4,5 , and 6 , depending on the point of origination of the call, as evaluated above. Therefore, the above expression approximates the intercluster handoff blocking probability.

Subject to the constraint that the total number of channels in $L_{\mathrm{Ak}}$ and $L_{\mathrm{Bk}}$ equals 62, all of the combinations of channel dimensions of $L_{\mathrm{Ak}}$ have been investigated to obtain the minimum value of $P_{B h 2}$ for $k=3,4,5$, and 6 at $\lambda_{o}=2300 / \mathrm{h} /$ cell, as shown in Fig. 15. For comparisons, the figure also includes simulation results of actual intercluster handoff blocking probabilities. It can be seen that the theoretical approximation underestimates the intercluster handoff blocking probabilities, given by the more accurate simulation results, by about $10 \%$. However, both theoretical estimates and simulation results indicate that to minimize the intercluster handoff blocking probability, there should be 61 channels assigned to link $L_{\mathrm{A} 3}, 53$ channels assigned to link $L_{\mathrm{A} 4}$, nine channels assigned to link $L_{\mathrm{A} 5}$, and 1 channel assigned to link $L_{\mathrm{A} 6}$ to minimize $P_{B h 2}$ at $\lambda_{o}=2300 / \mathrm{h} /$ cell. Table $\mathrm{V}$ shows the traffic parameters of Configuration II and the channel dimensions minimizing $P_{B h 2}$ at $1800 \leq \lambda_{\circ} \leq 2500 / \mathrm{h} /$ cell. Fig. 16 shows the new call blocking probabilities in cells 3 and 4 and the overall handoff blocking probability $P_{B h 2}$ with optimal channel dimensions at $1800 \leq \lambda_{\circ} \leq 2300 / \mathrm{h} /$ cell. Note that cell 4 has a higher new call blocking probability than cell 3 because of the smaller number of channels assigned to the direct link between cell 4 and MSC $A$ compared to cell 3 . The breaks in the theoretical curves of new call blocking probabilities for cells 3 and 4 in Configuration II are caused by changes in optimal channel dimensions relative to the call arrival rates, with one channel reassigned between crosslink and direct link at each breakpoint.

As discussed previously, more than 62 total channels can be assigned for partitioning between the direct and crosslinks to 
TABLE V

TRAFFiC PARAMETERS FOR CONFIGURATION II

\begin{tabular}{c|c|c|c|c|c}
\hline \multirow{2}{*}{$\begin{array}{c}\text { Connecting } \\
\text { links }\end{array}$} & Arrival rate & $\begin{array}{c}\text { Channel } \\
\text { holding time }\end{array}$ & \multicolumn{3}{|c}{ Channel dimensions to minimize $P_{B h 2}$} \\
\cline { 4 - 6 } & & $1800 \leq \lambda_{0} \leq 1900$ & $\lambda_{0}=2000$ & $2100 \leq \lambda_{0} \leq 2500$ \\
\hline $\mathrm{L}_{\mathrm{A} 3}, \mathrm{~L}_{\mathrm{B} 6}$ & $(1+\alpha+\delta) \lambda_{0}$ & $1 / \mu_{1}$ & 60 & 60 & 61 \\
\hline $\mathrm{L}_{\mathrm{A} 4}, \mathrm{~L}_{\mathrm{B} 5}$ & $(1+(2 / 3) \alpha) \lambda_{o}$ & $1 / \mu_{1}$ & 52 & 53 & 53 \\
\hline $\mathrm{L}_{\mathrm{A} 5}, \mathrm{~L}_{\mathrm{B} 4}$ & $(1 / 3)(\alpha+\beta) \lambda_{0}$ & $1 / \mu_{1}$ & 10 & 9 & 9 \\
\hline $\mathrm{L}_{\mathrm{A} 6}, \mathrm{~L}_{\mathrm{B} 3}$ & $(1 / 3)(3+\gamma) \lambda_{0}$ & $1 / \mu_{2}$ & 2 & 2 & 1 \\
\hline
\end{tabular}

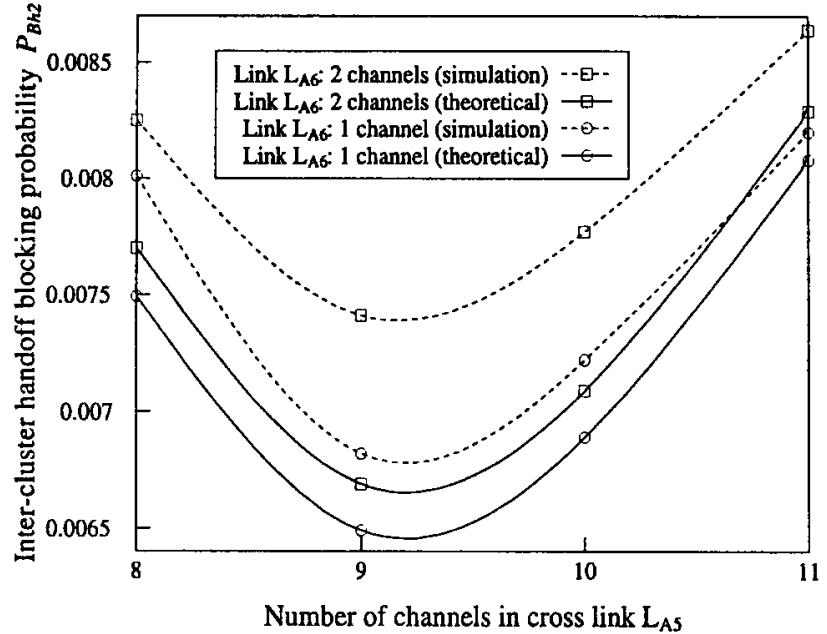

Fig. 15. Overall handoff blocking probability, $P_{B h 2}$, versus number of channels in $L_{\mathrm{A} 5}$ and $L_{\mathrm{A} 6}$ at $\lambda_{o}=2300 / \mathrm{h} /$ cell in Configuration II.

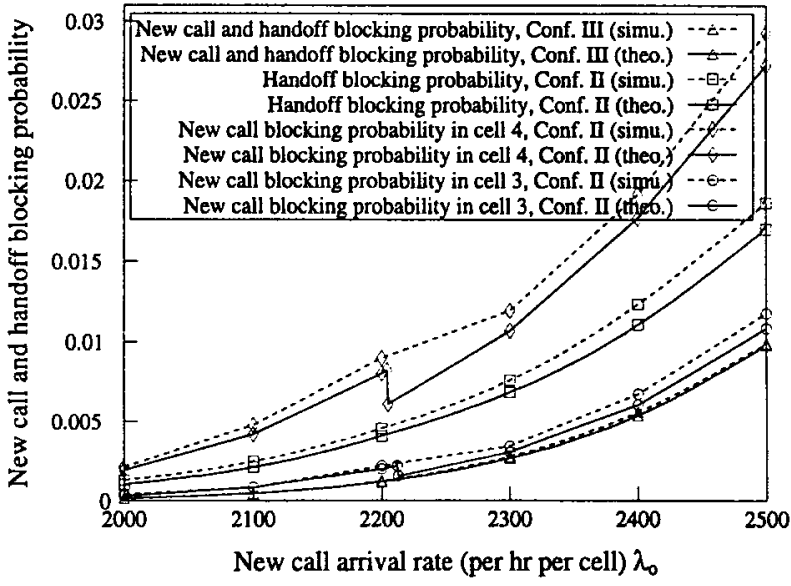

Fig. 16. New call and overall handoff blocking probabilities with optimal channel dimensions in Configurations II and III.

reduce the blocking probabilities. Fig. 11 shows the optimal dimensions of the crosslinks when they share more than 62 channels with the direct links. Fig. 17 shows the new call blocking probabilities in cells 3 and 4 and overall handoff blocking probabilities $P_{B h 2}$ when more than 62 channels are available between the direct and crosslinks. It can be seen from Fig. 17 that adding extra channels reduces the new call blocking probability for cell 4 significantly, the handoff blocking probability to a lesser extent, and the new call blocking probability for cell 3 imperceptibly. When the total number of channels is about 69 , all three probabilities are approximately equalized.
TABLE VI

Optimal Channel Dimensions in Configuration

II Versus Channel Holding Time and $\alpha$ with

$\beta=0.014, \gamma=0.001$, AND $P_{B n}<0.01$ IN CELL 4

\begin{tabular}{|c|c|c|c|c|c|c|c|c|}
\hline \multirow{2}{*}{\multicolumn{2}{|c|}{$\begin{array}{l}\mathrm{L}_{\mathrm{A} 3}, \mathrm{~L}_{\mathrm{A} 4} \\
\mathrm{~L}_{\mathrm{A} 5}, \mathrm{~L}_{\mathrm{A} 6} \\
\end{array}$}} & \multicolumn{7}{|c|}{ Channel holding time $1 / \mu_{l}$ (s) } \\
\hline & & \multirow{2}{*}{$\begin{array}{c}30 \\
60,55 \\
7,2\end{array}$} & \multirow{2}{*}{$\begin{array}{c}40 \\
60,55 \\
7,2\end{array}$} & \multirow{2}{*}{$\begin{array}{c}50 \\
60,55 \\
7,2\end{array}$} & \multirow{2}{*}{$\begin{array}{c}60 \\
60,55 \\
7,2\end{array}$} & \multirow{2}{*}{$\begin{array}{c}70 \\
60,55 \\
7,2\end{array}$} & \multirow{2}{*}{$\begin{array}{c}80 \\
61,55 \\
7,1\end{array}$} & \multirow{2}{*}{$\begin{array}{c}90 \\
61,55 \\
7,1\end{array}$} \\
\hline \multirow{7}{*}{ t } & 0.15 & & & & & & & \\
\hline & 0.20 & $\begin{array}{l}60,54 \\
8,2 \\
\end{array}$ & $\begin{array}{c}60,54 \\
8,2\end{array}$ & $\begin{array}{c}60,54 \\
8,2 \\
\end{array}$ & $\begin{array}{c}61,54 \\
8,1 \\
\end{array}$ & $\begin{array}{c}61,54 \\
8,1 \\
\end{array}$ & $\begin{array}{c}61,54 \\
8,1 \\
\end{array}$ & $\begin{array}{c}61,54 \\
8,1 \\
\end{array}$ \\
\hline & 0.25 & $\begin{array}{c}60,53 \\
9,2\end{array}$ & $\begin{array}{c}60,53 \\
9,2 \\
\end{array}$ & $\begin{array}{c}60,53 \\
9,2 \\
\end{array}$ & $\begin{array}{c}61,53 \\
9,1\end{array}$ & $\begin{array}{c}61,53 \\
9,1\end{array}$ & $\begin{array}{c}61,53 \\
9,1\end{array}$ & $\begin{array}{c}61,53 \\
9,1 \\
\end{array}$ \\
\hline & 0.30 & $\begin{array}{c}60,53 \\
9,2\end{array}$ & $\begin{array}{c}61,53 \\
9,1\end{array}$ & $\begin{array}{c}61,53 \\
9,1\end{array}$ & $\begin{array}{c}61,53 \\
9,1\end{array}$ & $\begin{array}{c}61,53 \\
9,1\end{array}$ & $\begin{array}{c}61,53 \\
9,1\end{array}$ & $\begin{array}{c}61,53 \\
9,1\end{array}$ \\
\hline & 0.35 & $\begin{array}{c}60,52 \\
10,2\end{array}$ & $\begin{array}{c}61,52 \\
10,1\end{array}$ & $\begin{array}{l}61,52 \\
10,1\end{array}$ & $\begin{array}{c}61,52 \\
10,1\end{array}$ & $\begin{array}{c}61,52 \\
10,1\end{array}$ & $\begin{array}{c}61,52 \\
10,1\end{array}$ & $\begin{array}{c}61,52 \\
10,1\end{array}$ \\
\hline & 0.40 & $\begin{array}{c}61,52 \\
10,1 \\
\end{array}$ & $\begin{array}{c}61,52 \\
10,1\end{array}$ & $\begin{array}{c}61,52 \\
10,1 \\
\end{array}$ & $\begin{array}{c}61,52 \\
10,1 \\
\end{array}$ & $\begin{array}{c}61,52 \\
10,1\end{array}$ & $\begin{array}{c}61,52 \\
10,1 \\
\end{array}$ & $\begin{array}{c}61,52 \\
10,1\end{array}$ \\
\hline & 0.45 & $\begin{array}{c}61,51 \\
11,1\end{array}$ & $\begin{array}{c}61,51 \\
11,1\end{array}$ & $\begin{array}{c}61,51 \\
11,1\end{array}$ & $\begin{array}{c}61,51 \\
11,1\end{array}$ & $\begin{array}{c}61,51 \\
11,1\end{array}$ & $\begin{array}{c}61,51 \\
11,1\end{array}$ & $\begin{array}{c}61,51 \\
11,1\end{array}$ \\
\hline
\end{tabular}

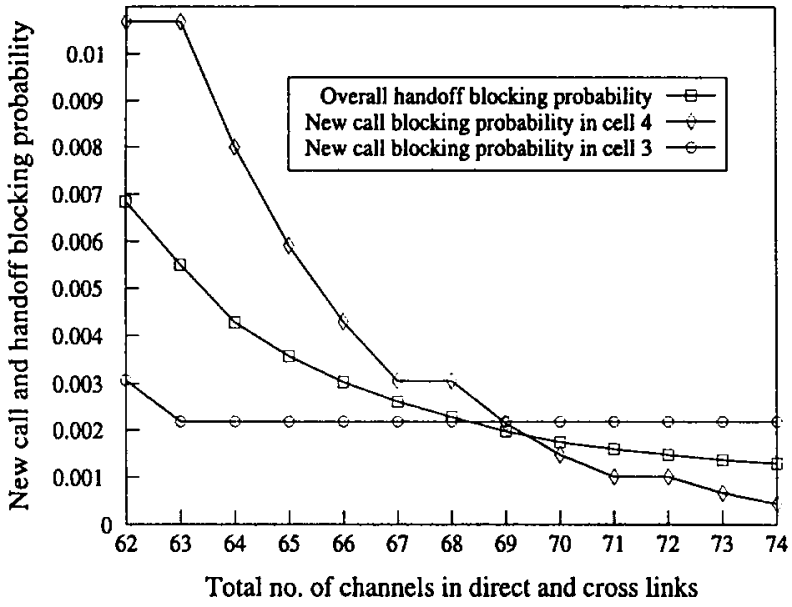

Fig. 17. New call and overall handoff blocking probability versus total number of channels in direct and crosslinks at $\lambda_{o}=2300 / \mathrm{h} /$ cell in Configuration II.

The optimal channel dimensions to minimize overall handoff blocking probability $P_{B h 2}$ for various channel holding times $1 / \mu_{1}$ and $\alpha$ have also been investigated. The values of $\beta$ and $\gamma$ are fixed to be 0.014 and 0.001 , respectively. Table VI shows the optimal channel dimensions under these conditions with $P_{B n}<0.01$. Whereas $L_{\mathrm{A} 6}$ requires one-two channels from high to low values of $1 / \mu_{1}$ and $\alpha$, the number of channels in $L_{\mathrm{A} 5}$ is relatively independent of $1 / \mu_{1}$ and increases as $\alpha$. Fig. 18 shows the maximum new call arrival rate $\lambda_{o}$ which can be attained. In comparison with Figs. 13 and 14, the maximum attainable new call arrival rate is slightly higher in Configuration II than in Configuration I because there are more crosslinks in Configuration II than in Configuration I. The handoff blocking probabilities $P_{B h 2}$ are between 0.0052 and 0.0065 , which is lower than those in channel assignment scheme $A$, but higher than those in channel assignment scheme $B$ of Configuration I.

\section{Link Capacity for Configuration III}

As shown in Fig. 4, the number of channels between the modified MSC's needs to be determined. With the mobility 


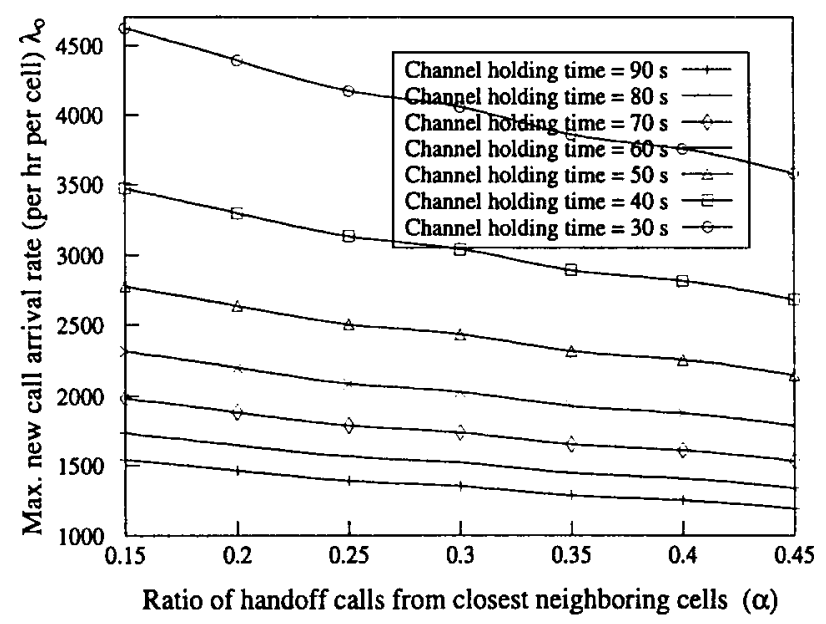

Fig. 18. Maximum new call arrival rate with $P_{B n}<0.01$ for optimal channel dimensions in Configuration II.

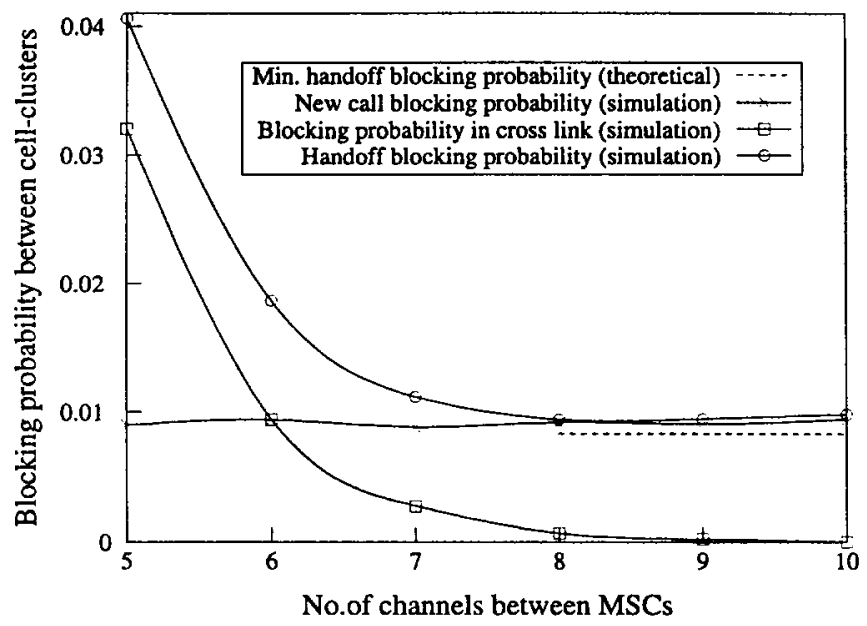

Fig. 19. Blocking probability between a pair of cells at the boundary of cell clusters versus number of channels between modified MSC's at $\lambda_{o}=2500 / \mathrm{h} /$ cell in Configuration III.

model described in Section III, computer simulations are employed to investigate the minimum number of channels required in link $L_{\mathrm{AB}}$ between the modified MSC's to attain the minimum handoff blocking probability. Fig. 19 shows the handoff blocking probability against the number of channels in link $L_{\mathrm{AB}}$ to handle handoff traffic between a single pair of cells at the cell-cluster boundary, employing the same handoff procedure as in scheme $A$ of Configuration I. It shows that eight channels in $L_{\mathrm{AB}}$ are sufficient to give a handoff blocking probability close to the minimum possible value contributed by the BSC-MSC links only, as discussed below. Fig. 20 shows the minimum number of channels required to carry handoff traffic between various numbers of pairs of cells at the cell-cluster boundary when the minimum handoff blocking probability is attained. The trunking efficiency of Configuration III is evident in this figure, as the number of channels required increases only 3.3 times when the number of cells at either side of the cell-cluster boundary is increased from one to six.

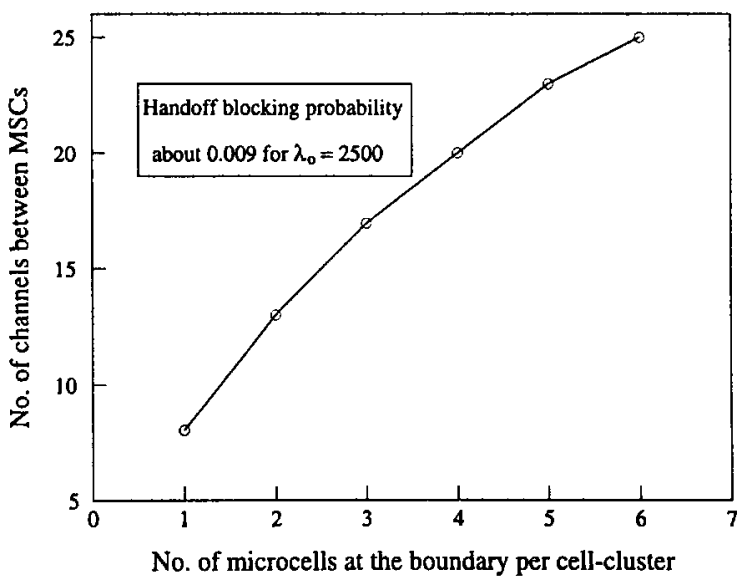

Fig. 20. Number of channels between modified MSC's versus number of pairs of cells at the boundary at $\lambda_{o}=2500 / \mathrm{h} /$ cell in Configuration III.

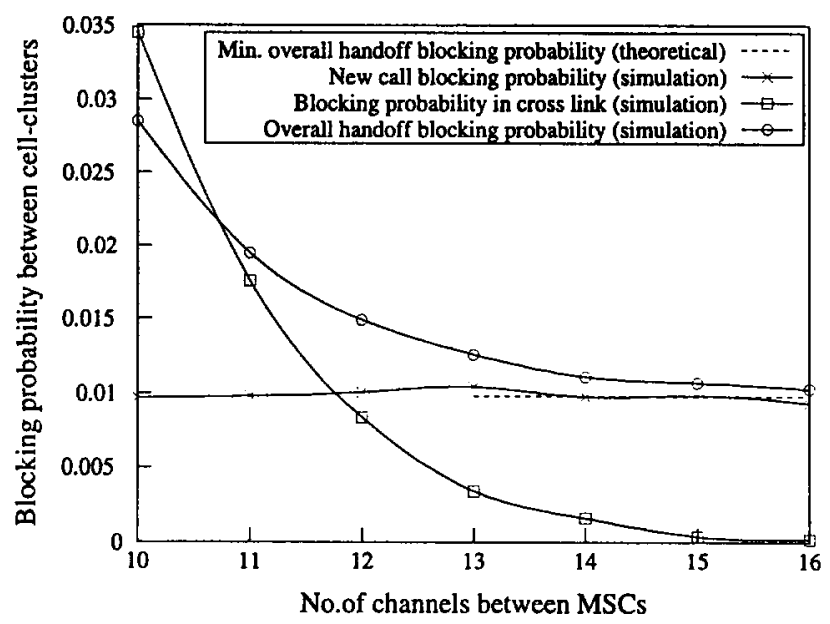

Fig. 21. Blocking probability of four cells at the boundary of cell clusters as arranged in Configuration II versus number of channels between modified MSC's at $\lambda_{o}=2500 / \mathrm{h} /$ cell in Configuration III.

Fig. 21 shows the handoff blocking probabilities with various numbers of channels in link $L_{\mathrm{AB}}$ when there are four cells at the boundary of the cell clusters involved in intercluster handoffs as in Configuration II. It shows that there should be 14 channels in the link connecting the modified MSC's in order to achieve a handoff blocking probability close to the minimum. The number of channels in link $L_{\mathrm{AB}}$ required to realize the handoff method in Configuration II is higher than that required to realize the handoff method in Configuration I because extra channels are needed for handoff calls in the inner region of the cell clusters.

In Figs. 19 and 21, the theoretical minimum handoff blocking probability is due to the blocking probability in the BS-MSC links (new call blocking probability), which is given by (3) with $m=62$ and call arrival rate $(1+\alpha) \lambda_{o}$. If the number of channels in $L_{\mathrm{AB}}$ is very large, the bottleneck for handoff call blocking will be due to the 62 channels in the BS-MSC links which are shared between new and handoff calls. The handoff blocking probability between cell clusters is close to this theoretical minimum when the number of channels between the modified MSC's is sufficiently large. 
Comparisons with Configurations I and II in Figs. 10 and 16 , respectively, show that the corresponding handoff method in Configuration III achieves lower new call and handoff blocking probabilities. For $P_{B n}<0.01$, Configuration III can accommodate new call arrivals at rate $\lambda_{0}=2500 / \mathrm{h} / \mathrm{cell}$, which is $14-25 \%$ higher than the corresponding values in Configurations I and II with the direct and crosslinks from each cell sharing 62 channels. On the whole, Configuration III is an improvement of Configurations I and II.

\section{CONCLUSIONS}

Three novel network configurations have been developed to provide seamless service for CDMA soft handoffs between cell clusters. Configurations I and II employ crosslinks to allow diversity combining of signals for soft handoffs. With a slight increase in complexity, Configuration II eliminates handoff oscillations between the two clusters when an MT moves back and forth across the cluster boundary. The optimal number of channels for each trunk group has been determined for these two configurations to minimize handoff blocking probabilities. Whereas Configurations I and II are compatible with the capability of existing MSC's to support CDMA soft handoffs, for Configuration III, the MSC's need to be modified so that they may be connected to each other to exchange diversity signals for combining in soft handoffs. In all cases, additional network intelligence is also required to enable network connection rerouting between MSC's. The optimal number of channels between the modified MSC's in Configuration III has been determined for the different handoff methods employed in Configurations I and II. The new call and handoff blocking probabilities in all configurations have been compared. The new call and handoff blocking probabilities for Configuration III are lower than the comparable case of Configurations I and II due to more efficient utilization of channel resources required to support soft handoffs. The tradeoff between the efficiency improvements and the increased complexity of the MSC's in Configuration III requires further investigations to economically justify its application. A method to trade off reductions in handoff blocking probability for increases in new call blocking probability has been considered as channel assignment scheme $B$ of Configuration I. While this method is also applicable to Configurations II and III, this has not been considered here due to space limitations.

\section{REFERENCES}

[1] W. C. Y. Lee, "Overview of cellular CDMA," IEEE Trans. Veh. Technol., vol. 40, pp. 291-302, May 1991.

[2] K. S. Gilhousen, I. M. Jacobs, R. Padovani, A. J. Viterbi, L. A. Weaver, Jr., and C. E. Wheatley, III, "On the capacity of a cellular CDMA system," IEEE Trans. Veh. Technol., vol. 40, pp. 303-312, May 1991.

[3] R. L. Pickholtz, L. B. Milstein, and D. L. Schilling, "Spread spectrum for mobile communications," IEEE Trans. Veh. Technol., vol. 40, pp. 313-322, May 1991.

[4] A. Salmasi and K. S. Gilhousen, "On the system design aspects of code division multiple access (CDMA) applied to digital cellular and personal communications networks," in Proc. VTC, 1991, pp. 57-62.
[5] C. M. Simmonds, D. Yang, and M. A. Beach, "Networking planning aspects of DS-CDMA systems with particular emphasis on soft handoff," in Antennas Propagat. (IEE Cong. Pub. 370), 1993, pp. 1007-1010.

[6] P. Seite, "Soft handoff in a DS-CDMA microcellular network," in Proc. VTC, 1994, pp. 530-534.

[7] S. C. Swales, J. C. S. Cheung, C. M. Simmonds, M. A. Beach, and J. P. McGeehan, "Handoff requirements for a third generation DS-CDMA air interface," in IEE Colloq. no. 151: Mobility in Support of Personal Communications, London, U.K., 1993, pp. 8/1-8/4.

[8] A. Baier, U. C. Fiebig, W. Granzow, W. Koch, P. Teder, and J. Thielecke, "Design study for a CDMA based third generation mobile radio system," IEEE J. Select. Areas Commun., vol. 12, pp. 733-743, May 1994.

[9] "An overview of the application of code division multiple access (CDMA) to digital cellular systems and personal cellular networks," Qualcomm Inc., Doc. EX60-10010, May 1992.

[10] A. S. Acampora and M. Naghshineh, "Control and quality of service provision in high speed microcellular networks," IEEE Personal Commun. Mag., vol. 1, pp. 36-43, 2nd quarter. 1994.

[11] _ "An architecture and methodology for mobile executed handoff in cellular ATM networks," IEEE J. Select. Areas Commun., vol. 12, pp. 1365-1375, Oct. 1994.

[12] O. T. W. Yu and V. C. M. Leung, "Connection architecture and protocols to support efficient handoffs over an ATM/B-ISDN personal communications network," ACM/Baltzer J. Mobile Networks Appl., vol. 1, no. 2, pp. 123-139, Oct. 1996.

[13] D. Hong and S. S. Rappaport, "Traffic model and performance analysis for cellular mobile radio telephone systems with prioritized and nonprioritized handoff procedures," IEEE Trans. Veh. Technol., vol. 35, no. 3, pp. 77-92, 1986

[14] M. M. Zonoozi, P. Dassanayake, and M. Faulkner, "Mobility modeling and channel holding time distribution in cellular mobile communication systems," in Proc. IEEE GLOBECOM, 1995, pp. 12-16.

[15] C. Jedrzycki and V. C. M. Leung, "Probability distribution of channel holding time in cellular telephony systems," in Proc. VTC, 1996, pp. 247-251.

[16] J. A. M. Nijhof, I. S. Dewantara, A. J. M. Roovers, and R. Prasad, "Base station system configurations for future universal mobile telecommunication systems," IEEE Trans. Veh. Technol., vol. 43, pp. 659-665, Aug. 1994.

[17] B. H. Cheung, "Network configuration for seamless support of CDMA soft handoffs between cell-clusters," Master's thesis, Univ. British Columbia, Canada, Dec. 1996

[18] W. C. Y. Lee, Mobile Cellular Telecommunications Systems, 2nd ed. New York: McGraw-Hill, 1995.

[19] K. K. Ho, "Architectural design of a code division multiple access cellular system," in Proc. VTC, 1992, pp. 47-50.

[20] J. R. Boucher, Traffic System Design Handbook. Piscataway, NJ: IEEE Press, 1993

[21] A. Murase, I. C. Symington, and E. Green, "Handover criterion for macro and microcellular systems," in Proc. VTC, 1991, pp. 524-530.

[22] D. Bertsekas and R. Gallager, Data Networks, 2nd ed. Englewood Cliffs, NJ: Prentice-Hall, 1992.

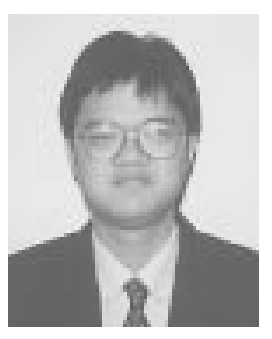

Bing H. Cheung was born in Hong Kong in 1971 $\mathrm{He}$ received the B.A.Sc. and M.A.Sc. degrees from the University of British Columbia, Vancouver, B.C., Canada, both in electrical engineering, in 1994 and 1997, respectively.

He is currently employed at Northern Telecom as a member of the Scientific Staff, specializing in radio resource management. His professional interests include multiple-access communications, mobile radio communications, traffic and performance analysis, and queueing theory.

Victor C. M. Leung (S'75-M'81), for a photograph and biography, see this issue, p. 1225. 